\title{
Tuning the brain for motherhood: prolactin-like central signalling in virgin, pregnant, and lactating female mice
}

Hugo Salais-López 1

Enrique Lanuza 2

Carmen Agustín-Pavón 1

Fernando Martínez-García 1,*

Phone +34964 387457

Emailfemartin@uji.es

1 Unitat Predepartamental de Medicina, Facultat de Ciències de la Salut, Universitat Jaume I, Av. de Vicent Sos Baynat, s/n, 12071 Castelló de la Plana, Spain

2 Departaments de Biologia Cel·lular i de Biologia Funcional, Facultat de Ciències Biològiques, Universitat de València, València, Spain

\section{Abstract}

Prolactin is fundamental for the expression of maternal behaviour. In virgin female rats, prolactin administered upon steroid hormone priming accelerates the onset of maternal care. By contrast, the role of prolactin in mice maternal behaviour remains unclear. This study aims at characterizing central prolactin activity patterns in female mice and their variation through pregnancy and lactation. This was revealed by immunoreactivity of phosphorylated (active) signal transducer and activator of transcription 5 (pSTAT5-ir), a key molecule in the signalling cascade of prolactin receptors. We also evaluated nonhypophyseal lactogenic activity during pregnancy by administering bromocriptine, which suppresses hypophyseal prolactin release. Late-pregnant and lactating females showed significantly increased pSTAT5-ir resulting in a widespread pattern of immunostaining with minor variations between pregnant and lactating animals, which comprises nuclei of the sociosexual and maternal brain, including telencephalic (septum, nucleus of the stria terminalis, and amygdala), hypothalamic (preoptic, paraventricular, supraoptic, and ventromedial), and midbrain (periaqueductal grey) regions. During late 
pregnancy, this pattern was not affected by the administration of bromocriptine, suggesting it to be elicited mostly by non-hypophyseal lactogenic agents, likely placental lactogens. Virgin females displayed, instead, a variable pattern of pSTAT5-ir restricted to a subset of the brain nuclei labelled in pregnant and lactating mice. A hormonal substitution experiment confirmed that estradiol and progesterone contribute to the variability found in virgin females. Our results reflect how the shaping of the maternal brain takes place prior to parturition and suggest that lactogenic agents are important candidates in the development of maternal behaviours already during pregnancy.

AQ1

AQ2

Keywords

Maternal care

Maternal aggression

Placental lactogens

pSTAT5

CD1 mice

Sociosexual brain

Abbreviations

$10 \mathrm{~N} \quad$ Dorsal motor nucleus of vagus

$12 \mathrm{~N} \quad$ Hypoglossal nucleus

3V Third ventricle

AAD Anterior amygdaloid area, dorsal part

AAV Anterior amygdaloid area, ventral part

AC Anterior commissural nucleus

aca Anterior commissure, anterior part

AcbC Accumbens nucleus, core

AcbSh Accumbens nucleus, shell

ACo Anterior cortical amygdaloid nucleus

acp Anterior commissure, posterior part

AD Anterodorsal thalamic nucleus

ADP Anterodorsal preoptic nucleus

AHA Anterior hypothalamic area, anterior part

AHC Anterior hypothalamic area, central part

AHiAL Amygdalohippocampal area, anterolateral part 
AHiPM Amygdalohippocampal area, post-eromedial part

AHP Anterior hypothalamic area, posterior part

AP Area postrema

APT Anterior pretectal nucleus

Aq Aqueduct (Sylvius)

Arc Arcuate hypothalamic nucleus

AVPe Anteroventral periventricular nucleus

BAC Bed nucleus of the anterior commissure

Bar Barrington's nucleus

BIC Nucleus of the brachium of the inferior colliculus

bic Brachium of the inferior colliculus

BLA Basolateral amygdaloid nucleus, anterior part

BLP Basolateral amygdaloid nucleus, posterior part

BLV Basolateral amygdaloid nucleus, ventral part

BMA Basomedial amygdaloid nucleus, anterior part

BMP Basomedial amygdaloid nucleus, posterior part

bp Brachium pontis

BSTIA Bed nucleus of the stria terminalis, intra-amygdaloid division

BSTLD Bed nucleus of the stria terminalis, lateral division, dorsal part

BSTLJ Bed nucleus of the stria terminalis, lateral division, juxtacapsular part

BSTLP Bed nucleus of the stria terminalis, lateral division, posterior part

BSTLV Bed nucleus of the stria terminalis, lateral division, ventral part

BSTMA Bed nucleus of the stria terminalis, medial division, anterior part

BSTMV Bed nucleus of the stria terminalis, medial division, ventral part

BSTMPI erointermediate part

BSTMPL Bed nucleus of the stria terminalis, medial division, post-erolateral part

BSTMPM Bed nucleus of the stria terminalis, medial division, post-eromedial part

cc Corpus callosum

$\mathrm{CC} \quad$ Central canal

$\mathrm{CeC} \quad$ Central amygdaloid nucleus, capsular part

$\mathrm{CeL} \quad$ Central amygdaloid nucleus, lateral division

CeM Central amygdaloid nucleus, medial division 
CeMAD Central amygdaloid nucleus, medial division, anterodorsal part

CeMAV Central amygdaloid nucleus, medial division, anterodorsal part

CeMPV Central amygdaloid nucleus, medial division, post-eroventral part

cg Cingulum

CI Caudal interstitial nucleus of the medial longitudinal fasciculum

Cir Circular nucleus

CLi Caudal linear nucleus of the raphe

CP Choroid plexus

cp Cerebral peduncle, basal part

$\mathrm{CPu} \quad$ Caudate putamen (striatum)

$\mathrm{Cu} \quad$ Cuneate nucleus

CxA Cortex-amygdala transition zone

D3V Dorsal third ventricle

Den Dorsal endopiriform nucleus

Dk Nucleus of Darkschewitsch

DLG Dorsal lateral geniculate nucleus

DLPAG Dorsolateral periaqueductal grey

DM Dorsomedial hypothalamic nucleus

DMPAG Dorsomedial periaqueductal grey

DMTg Dorsomedial tegmental area

DP Dorsal peduncular cortex

DpG Deep grey layer of the superior colliculus

DpMe Deep mesencephalic nucleus

DPO Dorsal periolivary region

DpWh Deep white layer of the superior colliculus

DR Dorsal raphe nucleus

DTgC Dorsal tegmental nucleus, central part

DTT Dorsal taenia tecta

eml External medullary lamina

EW Edinger-Westphal nucleus

f $\quad$ Fornix

fi Fimbria of the hippocampus

fmi Forceps minor of the corpus callosum

fr $\quad$ Fasciculus retroflexus

HDB Nucleus of the horizontal limb of the diagonal band 
Intercalated nuclei of the amygdala

ic

Internal capsule

IF

Interfascicular nucleus

IGL Intergeniculate leaf

IL Infralimbic cortex

InC Interstitial nucleus of Cajal

InCO Intercollicular nucleus

InG Intermediate grey layer of the superior colliculus

InWh Intermediate white layer of the superior colliculus

IP Interpeduncular nucleus

IPAC Interstitial nucleus of the posterior limb of the anterior commissure

IRt Intermediate reticular nucleus

La Lateral amygdaloid nucleus

LA Lateroanterior hypothalamic nucleus

LC Locus coeruleus

Ld Lambdoid septal zone

LD Laterodorsal thalamic nucleus

LDTg Laterodorsal tegmental nucleus

LGP Lateral globus pallidus

LH Lateral hypothalamic area

LHb Lateral habenular nucleus

$11 \quad$ Lateral lemiscus

lo Lateral olfactory tract

LOT Nucleus of the lateral olfactory tract

LP Lateral posterior thalamic nucleus

LPAG Lateral periaqueductal grey

LPBC Lateral parabrachial nucleus, central part

LPBD Lateral parabrachial nucleus, dorsal part

LPBE Lateral parabrachial nucleus, external part

LPBI Lateral parabrachial nucleus, internal part

LPBV Lateral parabrachial nucleus, ventral part

LPMR Lateral posterior thalamic nucleus, mediorostral part

LPO Lateral preoptic area

LRt Lateral reticular nucleus

LSD Lateral septal nucleus, dorsal part 
LSI Lateral septal nucleus, intermediate part

LSO Lateral superior olive

LSV Lateral septal nucleus, ventral part

LV Lateral ventricle

MCLH Magnocellular nucleus of the lateral hypothalamus

mcp Middle cerebellar peduncle

MCPC Magnocellular precommissural nucleus

MCPO Magnocellular preoptic nucleus

MdD Medullary reticular nucleus, dorsal part

MdV Medullary reticular nucleus, ventral part

ME Median eminence

MeA Medial amygdaloid nucleus, anterior part

MePD Medial amygdaloid nucleus, post-erodorsal part

MePV Medial amygdaloid nucleus, post-eroventral part

MGD Medial geniculate nucleus, dorsal part

MGM Medial geniculate nucleus, medial part

MGP Medial globus pallidus

MGV Medial geniculate nucleus, ventral part

MHb Medial habenular nucleus

ml Medial lemniscus

mlf Medial longitudinal fasciculus

MnPO Median preoptic nucleus

MnR Median raphe nucleus

MPA Medial preoptic area

MPB Medial parabrachial nucleus

MPBE Medial parabrachial nucleus, external part

MPO Medial preoptic nucleus

MS Medial septal nucleus

$\mathrm{mt} \quad$ Mammilothalamic tract

MTu Medial tuberal nucleus

MZMGV Marginal zone of the medial geniculate

ns Nigrostriatal bundle

opt Optic tract

OPT Olivary pretectal nucleus

OT Nucleus of the optic tract 
ox Optic chiasm

PAG Periaqueductal grey

PaLM Paraventricular nucleus, lateral magnocellular part

PaMM Paraventricular nucleus, medial magnocellular part

$\mathrm{PaPO} \quad$ Paraventricular nucleus, posterior part

$\mathrm{PaV} \quad$ Paraventricular nucleus, ventral part

PCom Nucleus of the posterior commissure

Pe Periventricular hypothalamic nucleus

PeF Perifornical nucleus

PF Parafascicular thalamic nucleus

$\mathrm{PH} \quad$ Posterior hypothalamic area

PIL Posterior-intralaminar thalamic nucleus

Pir Piriform cortex

PLCo Post-erolateral cortical amygdaloid nucleus

PM Paramedian lobule

PMD Premammillary nucleus, dorsal part

PMV Premammillary nucleus, ventral part

PnC Pontine reticular nucleus, caudal part

$\mathrm{PnO} \quad$ Pontine reticular nucleus, oral part

PnR Pontine raphe nucleus

PnV Pontine reticular nucleus, ventral part

PoT Posterior thalamic nuclear group, triangular part

PP Peripeduncular nucleus

PPT Posterior pretectal nucleus

PPTg Pedunculopontine tegmental nucleus

Pr5 Principal sensory trigeminal nucleus

PSTh Parasubthalamic nucleus

PV Paraventricular thalamic nucleus

PVA Paraventricular thalamic nucleus, anterior part

py Pyramidal tract

R Red nucleus

$\mathrm{RCh} \quad$ Retrochiasmatic area

$\mathrm{Re} \quad$ Reuniens thalamic nucleus

RMg Raphe magnus nucleus

Rob Raphe obscurus nucleus 
Rubrospinal tract

$\mathrm{Rt}$

Reticular thalamic nucleus

s5

Sensory root of the trigeminal nerve

SCh Suprachiasmatic nucleus

SCO Subcommissural organ

scp Superior cerebellar peduncle

SFi Septofimbrial nucleus

SFO Subfornical organ

SG Suprageniculate thalamic nucleus

SHi Septohippocampal nucleus

SI Substantia innominata

SLEA Sublenticular extended amygdala

sm Stria medullaris of the thalamus

SN Substantia nigra

SNC Substantia nigra, pars compacta

SNR Substantia nigra, reticular part

SO Supraoptic nucleus

sol Solitary tract

Sol Nucleus of the solitary tract

SolC Nucleus of the solitary tract, commissural part

SolDL Nucleus of the solitary tract, dorsolateral part

SolG Nucleus of the solitary tract, gelatinous part

SolM Nucleus of the solitary tract, medial part

SolV Nucleus of the solitary tract, ventral part

Sp5 Spinal trigeminal nucleus

Spa Subparaventricular zone of the hypothalamus

SPFPC Subparafascicular thalamic nucleus, parvicellular part

SPO Superior paraolivary nucleus

st Stria terminalis

STh Subthalamic nucleus

str Superior thalamic radiation

SubB Subbrachial nucleus

SubCD Subcoeruleus nucleus, dorsal part

SubCV Subcoeruleus nucleus, ventral part

SubG Subgeniculate nucleus

SubI Subincertal nucleus 
SuMM Supramammillary nucleus, medial part

TC Tuber cinereum

Te Terete hypothalamic nucleus

$\mathrm{Tu} \quad$ Olfactory tubercle

VEn Ventral endopiriform nucleus

VLG Ventral lateral geniculate nucleus

VLGMC Ventral lateral geniculate nucleus, magnocellular part

VLGPC Ventral lateral geniculate nucleus, parvicellular part

VLPAG Ventrolateral periaqueductal grey

VLPO Ventrolateral preoptic nucleus

VMH Ventromedial hypothalamic nucleus

VMHc Ventromedial hypothalamic nucleus, central part

VMHdm Ventromedial hypothalamic nucleus, dorsomedial part

VMHvl Ventromedial hypothalamic nucleus, ventrolateral part

VMPO Ventromedial preoptic nucleus

VOLT Vascular organ of the lamina terminalis

VP Ventral pallidum

VRe Ventral reuniens thalamic nucleus

vsc Ventral spinocerebellar tract

VTA Ventral tegmental area

ZI Zona incerta

ZID Zona incerta, dorsal part

ZIV Zona incerta, ventral part

Electronic supplementary material

The online version of this article (doi:10.1007/s00429-016-1254-5) contains supplementary material, which is available to authorized users.

\section{Introduction}

Prolactin (PRL) is an adenohypophyseal hormone known for participating in the development of the mammary gland and the production of milk. Aside from this, PRL exerts multiple actions in the brain to adapt the female physiology and behaviour to the challenge of motherhood. For example, PRL is involved in suppressing fertility, increasing resilience to stress, and adjusting energy and body fluid regulation during lactation (Grattan et al. 2001). One of the most important roles of PRL is the development of a number of behaviours aimed at 
ensuring the survival and welfare of the offspring, namely maternal behaviours (Bridges 1994). In rodents, these behaviours include nest building, maternal care of pups (grooming, nurturing and hovering over them), and their defence against potentially infanticide intruders, i.e., maternal aggression (Lonstein and Gammie 2002; Numan and Insel 2003).

However, the exact role of prolactin in the expression of maternal behaviours is still unclear. In rats, virgin females are neophobic towards pups and only engage in allomaternal care after a 1-week period of exposure to the young (Fleming and Rosenblatt 1974). This so-called "maternal sensitisation" can be significantly shortened by means of a hormonal treatment mimicking the endocrine events of the peri-partum period, consisting in the administration of PRL on top of the appropriate gonadal steroid profile (Bridges et al. 1990; Bridges and Ronsheim 1990). Employing a similar approach, Mayer et al. (1990) induced maternal-like aggression in virgin female rats. This evidence strongly suggests that the development of maternal behaviours in the rat is facilitated by lactogenic signals in a synergistic action with gonadal steroids.

In contrast to rats, virgin female mice display almost spontaneous allomaternal care, as they usually retrieve and take care of pups in a short time during their first exposure to them (a few minutes, see Numan and Insel 2003 ) with no apparent need of maternal sensitisation (Stolzenberg and Rissman 2011; MartínSánchez et al. 2015b). This suggests that maternal care in mice is independent of endocrine factors, e.g., PRL. In this line, it has been proposed that pup-derived stimuli, such as nipple stimulation (Garland and Svare 1988; Stern and Kolunie 1993 ), olfactory (Wang and Storm 2011; Weiss et al. 2011), and/or vomeronasal cues (Leypold et al. 2002; Hasen and Gammie 2009), might be important in the development of maternal care and maternal aggression. However, disruption of PRL signalling through the deletion of the PRL receptor gene severely impairs several forms of maternal care (Lucas et al. 1998). Furthermore, some components of maternal behaviour still appear to need of endocrine agents related to pregnancy and lactation to be properly expressed. Maternal aggression, for example, is not performed spontaneously by virgin female mice, so otherwise fully maternal virgins do not attack male conspecifics even when in close and continuous contact with pups (Martín-Sánchez et al. 2015a). Altogether, this evidence supports a putative role of PRL in the development of maternal behaviours, as part of the endocrine milieu of pregnancy and lactation. Still, the exact period and sites in the female brain where PRL exerts these adaptive changes remain unknown.

To gain more insight into the actions of PRL in the shaping of the maternal brain, it would be of great help to obtain a complete picture of the central 
activity of PRL throughout the reproductive cycle of the female mouse, including the basal state (virgins), pregnancy, and lactation. A valuable indicator of lactogenic activity in the brain is the level of signalling occurring through the PRL receptor (PRLR). The PRLR belongs to the type I cytokine receptor superfamily and counts with different variants generated by alternative splicing, termed either long (PRLR-1) or short (PRLR-s) according to the length of its intracellular domain and docking specificity (Freeman et al. 2000). Mice, for example, have one long and three different short isoforms (Freeman et al. 2000). Functional PRL signalling occurs mainly through the PRLR-1, whereas short variants of the receptor have no clear functionality (Lesueur et al. 1991). Activation of the PRLR-1 by PRL predominately triggers the JAK/STAT pathway (Binart et al. 2010), leading to phosphorylation (activation) of Signal Transducer and Activator of Transcription 5 (pSTAT5), which eventually translocates to the nucleus to mediate the biological effects of PRL (Bole-feysot et al. 1998). In the brain, for example, phosphorylation of STAT5 has been linked to the negative feedback control of PRL release in the tuberoinfundibular dopaminergic (TIDA) hypothalamic neurons (Ma et al. 2005). Hence, the specific detection of pSTAT5 serves as a functional indicator of signalling through the PRLR-1, consequently, highlighting the main component of lactogenic activity (Brown et al. 2010, 2011).

Importantly, hypophyseal PRL is not the only lactogenic agent operating through the PRLR-1. During the second half of pregnancy, for example, the placenta produces the so-called "placental lactogens" (PLs), PRL homologues that also bind the PRLR (Soares et al. 1998) and promote maternal behaviours in the rat (Bridges et al. 1996). The potential contribution of secondary lactogenic sources, such as PLs, should be evaluated during their critical period of action, i.e., pregnancy.

In the present study, we sought to characterise the anatomical patterns of PRLlike signalling in the brain of virgin, late-pregnant, and lactating female mice. To do so, we employed the immunohistochemical detection of pSTAT5 as a reporter of PRLR activation. First, we mapped the general pSTAT5 immunoreactivity (pSTAT5-ir) in the brain of virgin, pregnant, and lactating mice. We also assessed the density of immunoreactive cells in selected brain regions and compared the levels of pSTAT5-ir of our experimental groups. An additional group of pregnant animals treated with bromocriptine was included in the analysis to evaluate the putative central action of non-hypophyseal lactogenic agents during pregnancy. Bromocriptine is a dopamine D2-receptor agonist that selectively suppresses the production of hypophyseal PRL by emulating the release of dopamine by the TIDA neurons, as part of the negative feedback control of PRL secretion (Bridges and Ronsheim 1990). 
Given the variability in the distribution of pSTAT5-ir in the brain of virgin, freely cycling females, we also explored the contribution of gonadal steroids to brain lactogenic signalling. For this purpose, we analysed the levels of pSTAT5ir in ovariectomized virgin female mice that received treatment with estradiol or estradiol plus progesterone (hormonal substitution) plus a control group.

By assessing the basal profile of lactogenic signalling in female mice and how it changes with the physiological events of pregnancy and lactation, we aim at clarifying the role of lactogenic signals in the management of maternal behaviours.

\section{Materials and methods}

\section{Animals}

For the present study, adult female CD1 mice (Charles River Laboratories, France), 8-12 weeks of age $(n=49)$, were group housed (4-6 animals per group) in polypropylene plastic cages under the controlled temperature $\left(24 \pm 2{ }^{\circ} \mathrm{C}\right)$ and lighting conditions ( $12 \mathrm{~h}: 12 \mathrm{~h}$; lights $\mathrm{ON}$ at $8 \mathrm{am}$ ), with ad libitum access to food and water. Animals were treated throughout according to the European Union Council Directive of June 3, 2010 (6106/1/10 REV1), and procedures were approved by the Committee of Ethics on Animal Experimentation of the University of Valencia, where the experiments were performed.

\section{Experimental design}

Mapping of pSTAT5-ir patterns in virgin, late-pregnant, and lactating mice

Animals $(n=27)$ were randomly assigned to a total of four groups: (1) virgin, freely cycling females $(n=7)$; (2) late-pregnant females, post-conception day (PD) 14-18 ( $n=6)$; (3) late-pregnant females, PD 14-18, treated with bromocriptine $(n=8)$, and (4) lactating dams on post-partum day 6 (PPD6) $(n=6)$. Treatment for bromocriptine group consisted in two sequential, s.c., injections of $100 \mu \mathrm{g}$ of bromocriptine (2-bromo- $\alpha$-ergocriptine, Sigma-Aldrich, St. Louis, USA) in $10 \% \mathrm{EtOH}, 19$ and $2 \mathrm{~h}$ prior to perfusion (Sjoeholm et al. 2011 ). This resulted in a dose of $2 \mathrm{mg} / \mathrm{kg}$ of body weight per injection. A similar bromocriptine treatment schedule abolished pSTAT5-ir in some structures of the brain of freely cycling virgin female mice, whereas some residual, strongly reduced labelling was observed in other nuclei. When compared with non-treated females, bromocriptine treatment resulted in an overall reduction of pSTAT5-ir higher than $75 \%$ (data not shown). This demonstrates an effective inhibition of hypophyseal release of PRL by the bromocriptine treatment. 
To ensure mating in groups 2,3 , and 4 , females of these groups were paired with a stud male for a period of 4 days, counting the first day as pregnancy day 1 (PD1). Following impregnation, females were housed in pairs until the day of perfusion. Females of pregnant and bromocriptine groups were perfused on presumed PD18, but the exact pregnancy day was assessed post-mortem, evaluating the developmental stage of the respective foetuses through anatomical signs, such as the differentiation of the eyelids, tongue, whiskers, or fingers (http://www.emouseatlas.org).

\section{Hormonal substitution in virgin female mice}

For this experiment, females $(n=18)$ underwent ovariectomy under, i.p., ketamine (Imalgene 500, Merial, Toulouse, France, $75 \mathrm{mg} / \mathrm{kg}$ ) and medetomidine (Domtor $1 \mathrm{mg} / \mathrm{ml}$, Esteve, Barcelona, Spain, $1 \mathrm{mg} / \mathrm{kg}$ ) anaesthesia. Females were also given butorphanol tartrate $1 \%$ (Torbugesic, Fort Dodge, Girona, Spain, $20 \mu 1$ s.c.) for pain control and sedation and atipamezole (Antisedan, Esteve, Barcelona, Spain, $1 \mathrm{mg} / \mathrm{kg}$ i.p.) after surgery, to facilitate awakening and restoration. After 7-10 days of recovery, females were randomly assigned to three experimental groups, for subsequent hormonal treatments: (1) control group received two injections of vehicle (sunflower oil; OO, $n=6$ ); (2) estradiol + oil group (EO, $n=6$ ); and (3) estradiol + progesterone group (EP, $n=6)$. In accordance with the experimental induction of the estrous cycle (Rissman et al. 1997), estradiol was administered on a slow-release profile throughout the experiment by means of the subcutaneous placement of silastic tubing implants (Dow Corning Corporation, 1.57-mm inner diameter, 2.41-mm outer diameter). Silastic implants measured $20 \mathrm{~mm}$ and were filled with $20 \mu \mathrm{g} / \mathrm{ml} \beta$-estradiol (Sigma, St. Louis, MO, USA) in sunflower oil. By contrast, progesterone (Sigma, St. Louis, MO, USA) was administered acutely in a 500- $\mu \mathrm{g}$ subcutaneous injection, diluted in sunflower oil. Estradiol (or vehicle) implants were applied in the morning of experimental day 1 (D1). Six days later, on the morning of D7, females were given an acute dose of progesterone (or vehicle). Three hours later, every animal was given a $5 \mathrm{mg} / \mathrm{kg}$, i.p., dose of ovine PRL (Sigma, St. Louis, MO, USA) and perfused 45 min later (Brown et al. 2010). This PRL administration renders homogeneous supraphysiological levels of circulating PRL, thus allowing evaluation of steroid influence on PRL signalling, excluding the effects of estradiol on hypophyseal PRL release (Maeda et al. $1996)$.

\section{AQ3}

\section{Histological processing and pSTAT5 immunohistochemistry}

Animals were given an overdose of pentobarbital and perfused transcardially 
with $4 \%$ paraformaldehyde in 0.1-M phosphate buffer (PB) $\mathrm{pH}$ 7.4. Brains were carefully extracted and post-fixed overnight in the same fixative, then cryoprotected in $30 \%$ sucrose in $0.01-\mathrm{M} \mathrm{PB}$ until they sank. Five parallel series of 40- $\mu \mathrm{m}$-thick coronal sections were obtained with a freezing microtome (Microm HM-450, Walldorf, Germany) and stored in PB-30 \% sucrose at $-20{ }^{\circ} \mathrm{C}$.

Immunohistochemistry was conducted in free-floating sections under light shaking at room temperature $\left(25^{\circ} \mathrm{C}\right)$ unless otherwise stated. Immunohistochemistry protocol was adapted from Brown et al. $(2010,2011)$. Briefly, sections were thoroughly rinsed between stages for at least three 10-min washes in TRIS-buffered saline, $0.05 \mathrm{M}, \mathrm{pH} 7.6$ (TBS). After thawing, sections underwent an initial antigen retrieval step, consisting in two sequential 6-min incubations in $0.01-\mathrm{M}$ TRIS buffer (TB), $\mathrm{pH} 10$ at $85^{\circ} \mathrm{C}$, and brought quickly to room temperature in between. Tissue was then incubated in (a) $1 \%$ hydrogen peroxide $\left(\mathrm{H}_{2} \mathrm{O}_{2}\right)$ for $30 \mathrm{~min}$, (b) $2 \% \mathrm{BSA}, 2 \%$ goat serum and $0.3 \%$ Triton X100 in TBS for $1 \mathrm{~h}$, (c) rabbit anti-pSTAT5 primary antibody (pSTAT5 Tyr694; Cell Signalling Technology, Beverly, MA, USA) diluted 1:500 in TBS plus Triton X-100 $0.1 \%$ for $72 \mathrm{~h}$ at $4{ }^{\circ} \mathrm{C}$, (d) biotinylated goat anti-rabbit IgG (Vector Laboratories, Peterborough, UK) 1:200 in TBS for 90 min, and (e) avidin-biotinperoxidase complex (ABC Elite kit; Vector Laboratories) in TBS for $90 \mathrm{~min}$. Peroxidase label was developed using $0.005 \%$ 3-3'-diaminobenzidine (Sigma) and $0.01 \% \mathrm{H}_{2} \mathrm{O}_{2}$ in $\mathrm{TB} \mathrm{pH} 7.6$ for about $15 \mathrm{~min}$, obtaining thereby a brown nuclear staining. Sections were rinsed in TB and mounted onto gelatinized slides, dehydrated in alcohols, cleared with xylene, and coverslipped with Entellan.

\section{Image acquisition and quantitative assessment of pSTAT immunoreactivity}

To quantify the levels of PRL signalling, we analysed the density of cells showing pSTAT5 immunoreactivity (pSTAT5-ir) in different nuclei of the sociosexual brain, and other nuclei involved in the regulation of maternal behaviours (see "Results"). To do so, we selected representative frames of the chosen nuclei (see "Results") using the stereotaxic atlas of Paxinos and Franklin (2004), and we obtained photomicrographs of these frames in both hemispheres using a digital camera (Leica DFC495) attached to a microscope Leitz DMRB (Leica AG, Germany). Image processing and analysis were conducted on Image J. Briefly, we subtracted background light and converted the RGB colour image to greyscale by selecting the green channel. Then, we binarised the greyscale image, setting the $75 \%$ of the mode of the histogram as a threshold, thus, including every pixel below this threshold as immunolabelled. We filtered 
smaller noise particles by an additional processing consisting of the following Image J commands: "fill holes"; "open" (three iterations) and "watershed". Particles were additionally filtered by area (larger than $70 \mu \mathrm{m}^{2}$, corresponding to an approximate diameter of $9.4 \mu \mathrm{m}$ ) and finally counted automatically by Image J. We calculated the mean (interhemispheric) density of pSTAT5immunoreactive cell nuclei for each specimen by dividing the mean value of these counts for both hemispheres by the area of the respective frame.

We conducted statistical analysis of the resulting data on the SPSS software package. After checking for normality (Kolmogorov-Smirnov test with Lilliefors' correction) and homogeneity of variance (Levene's test), we performed a separate one-way ANOVA test on each of the analysed brain regions to assess inter-group differences (virgin, pregnant, bromocriptine-treated pregnant, and lactating) on the mean pSTAT5-ir density. Statistically significant differences $(p \leq 0.05)$ were further explored by means of post-hoc pairwise comparisons with Bonferroni's correction.

\section{Results}

The immunohistochemical detection of pSTAT5 rendered a clear nuclear staining in the brain tissue (Fig. 1). We found apparent differences in the immunostaining patterns between late-pregnant and lactating females, on the one hand, and virgin females, on the other. Whereas pregnant and lactating females showed a homogenous pattern of pSTAT5-ir distribution with minor interindividual qualitative differences, virgin females displayed variable immunolabelling patterns. In this section, we first describe the pattern of pSTAT5 -ir in the brain of pregnant/lactating females, and then, we briefly illustrate the variable immunostaining observed in virgin females (see Figs. 1, 2). Finally, we report the results of a quantitative analysis comparing the density of pSTAT5-ir in a subset of functionally relevant nuclei in all four experimental groups. For the description of the results, we follow the neuroanatomical terminology and abbreviations by Franklin and Paxinos (see Abbreviations list).

\section{Fig. 1}

Appearance of pSTAT5 immunoreactivity in the brains of late-pregnant, lactating, and virgin female mice. Photomicrographs showing the pattern of pSTAT5 immunoreactivity of the BST-MPA region in a a post-conception day 14-pregnant female; $\mathbf{b}$ a day 14 pregnant female treated with bromocriptine; $\mathbf{c}$ a post-partum day 6 lactating dam and three different virgin females with different staining densities $(\mathbf{d}-\mathbf{f})$. Immunohistochemistry for pSTAT5 resulted in a specific nuclear labelling (arrows on inset $\mathbf{a}^{\prime}$ ). The pattern of pSTAT5-ir was constant among latepregnant and lactating females $(\mathbf{a}-\mathbf{c})$, with a high number of tightly packed 
immunostained cells in the BST-MPA continuum. Conversely, the pattern of pSTAT5 immunostaining in virgin females showed a remarkable inter-individual quantitative variability, ranging from barely no staining (d), a moderate staining density (e), to an exceptionally high density similar to that in late-pregnant females and dams (f). Every section is approximately $0.25 \mathrm{~mm}$ posterior to bregma. Scale bars $250 \mu \mathrm{m} ; 50 \mu \mathrm{m}$ (inset)
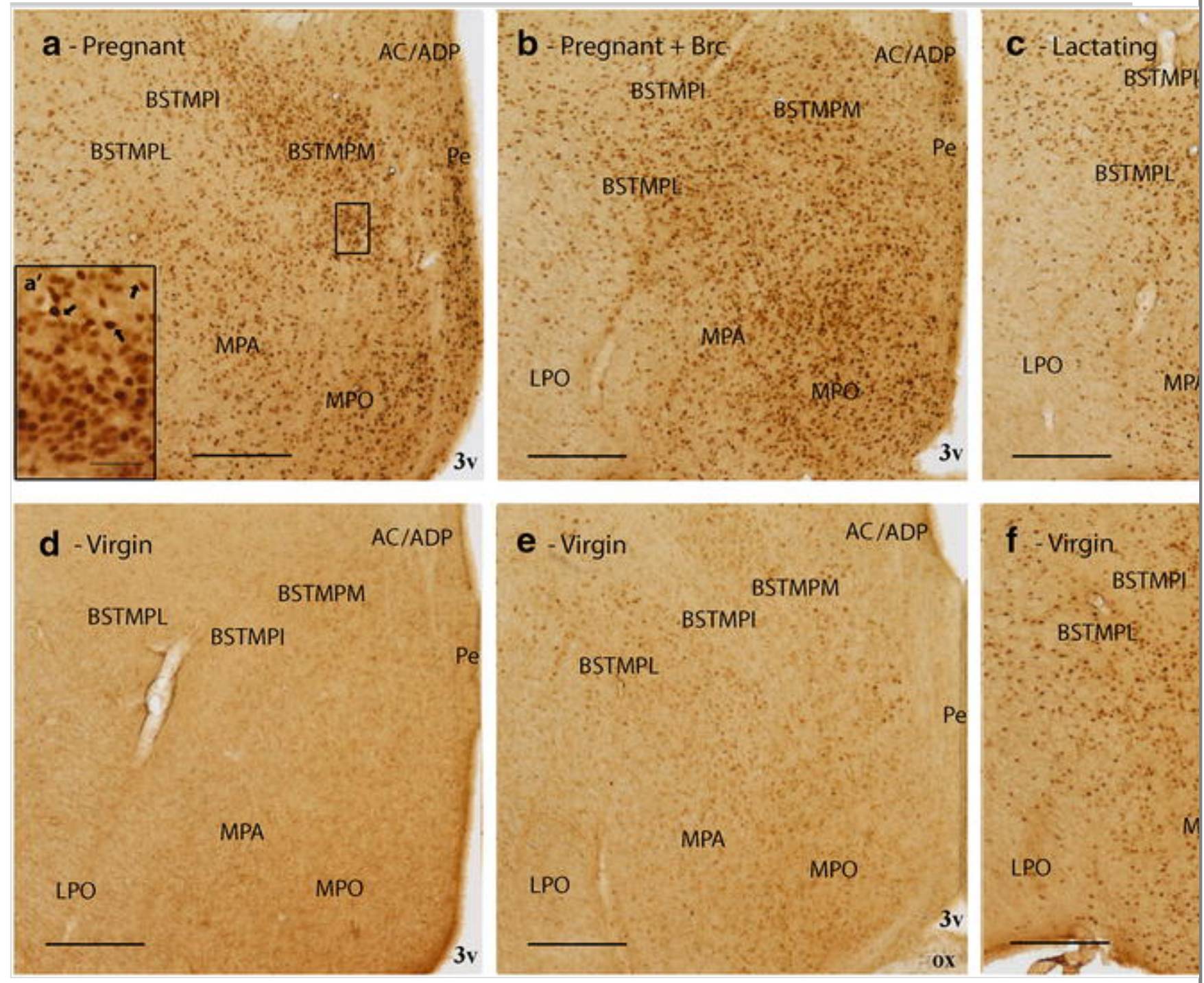

LPO

Fig. 2

Distribution of pSTAT5 immunoreactivity in the brains of late-pregnant, lactating, and virgin female mice. Semi-schematic camera-lucida drawings of coronal sections through the brain of a post-conception day 14-pregnant female (specimen M1392) treated with bromocriptine before perfusion (see text). Each red dot represents 3-4 immunoreactive cells. Since the distribution and density of immunostained cells were similar in other late-pregnant and lactating females, this figure illustrates the canonical pattern of pSTAT5-ir in the brain of both groups of females. The background colours in the sections encode a frequency score for the presence of pSTAT5 immunostained cells in the brain of virgin females: no pSTAT5-ir in any virgin female (white); pSTAT5-ir in 1-2 specimens (blue); 
pSTAT5 in 3-5 specimens (pink); and pSTAT5-ir in 6-7 animals (orange). Approximate distance to bregma is indicated for each section

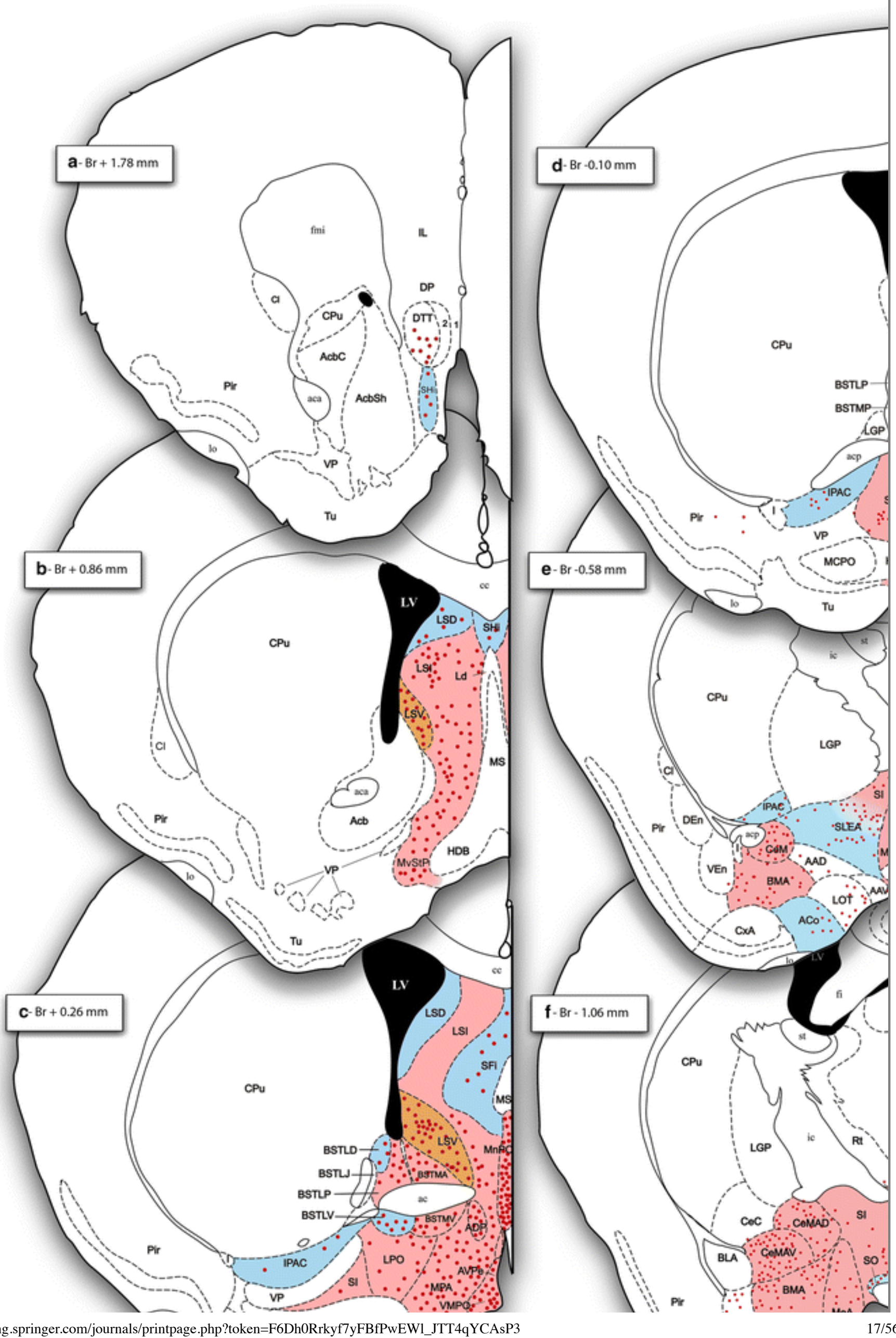



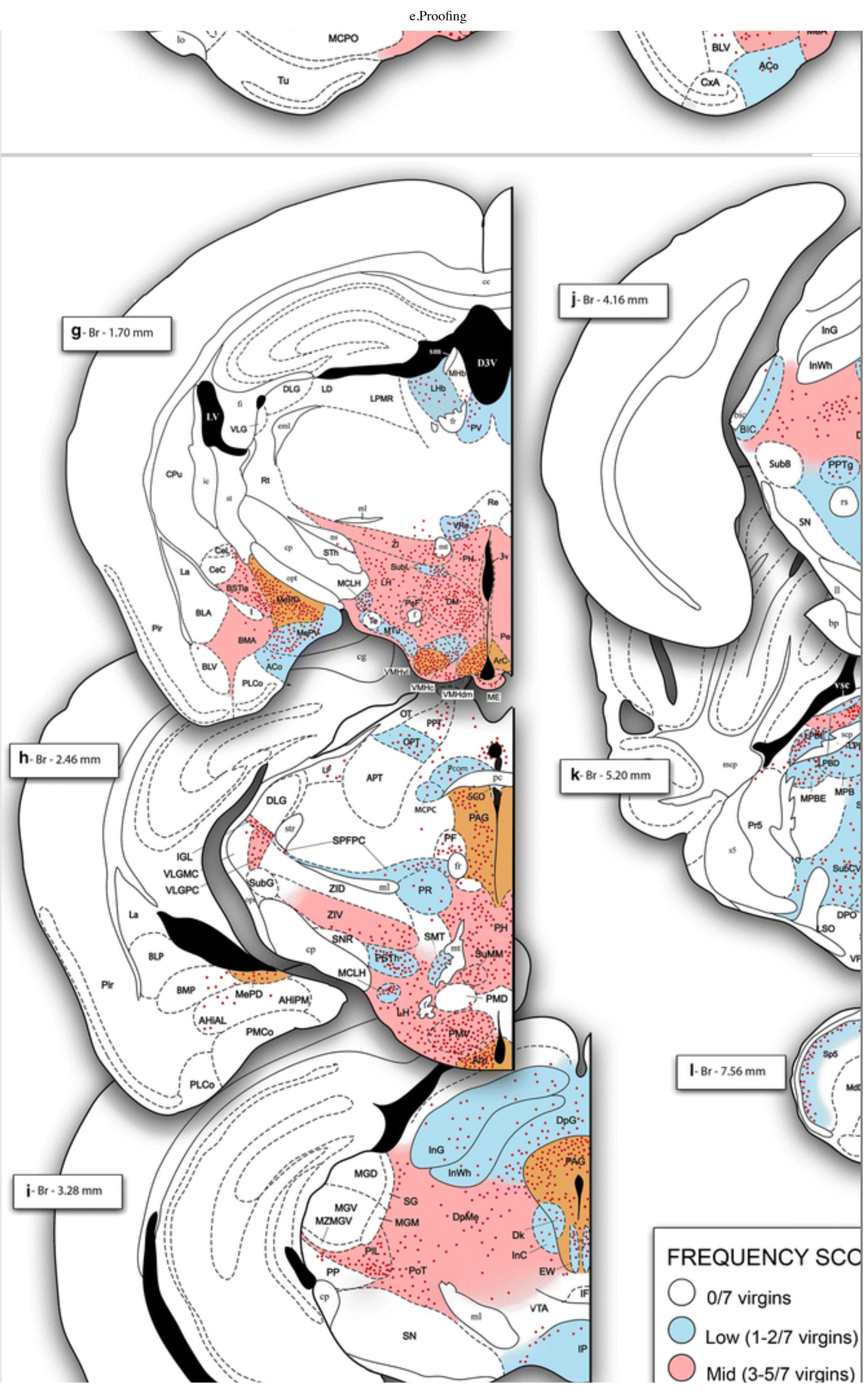


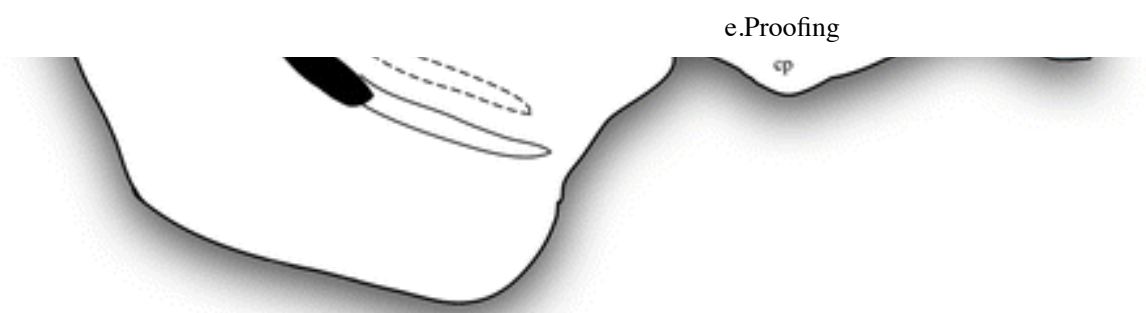

\section{Pattern of pSTAT5 Immunoreactivity in the brain of pregnant/lactating female mice}

A close analysis of the immunoreactivity for pSTAT5 in these females indicates no apparent qualitative differences between both groups, with very few exceptions (see below). In addition, treatment with bromocriptine during late pregnancy had no visible qualitative effect on the distribution of pSTAT5-ir. Therefore, we first describe the common pattern of distribution of pSTAT5-ir in all three experimental groups under the common pattern shared by late-pregnant and lactating females.

Importantly, the choroid plexus showed some degree of immunostaining in every animal, regardless of physiological state (not shown). This is likely related to their role in PRL transport to the brain (Mangurian et al. 1992; but see Brown et al. 2015 ). In addition, in pregnant/lactating females, quite abundant cell labelling was present in the cerebral hemispheres (septum, amygdala, extended amygdala, and, to a lesser extent, cortex), diencephalon (mainly in the hypothalamus, but also in some thalamic and pretectal nuclei), midbrain (periaqueductal grey and laterodorsal tegmentum) and hindbrain.

\section{Telencephalon}

Immunolabelling for pSTAT5 in this major brain division was present mainly in subpallial structures, but also appeared in a small number of pallial areas. Within the pallial telencephalon, neither the neocortical region nor the hippocampal formation showed pSTAT5-ir. As for the olfactory cortical areas, the olfactory bulbs were also devoid of pSTAT5-ir, whereas immunolabelled cells were present in the dorsal tenia tecta (DTT, Fig. 2 a). Furthermore, several nuclei in the cortical amygdala contained pSTAT5-ir, namely, the nucleus of the lateral olfactory tract (LOT, Fig. 2 e), the anterior cortical amygdaloid nucleus (ACo, Fig. 2e, f), the basomedial amygdaloid nucleus (BMA and BMP, Fig. $2 \mathrm{~g}, \mathrm{~h}$ ), the ventral basolateral amygdaloid nucleus (BLV, Fig. $2 \mathrm{f}$ ), and the amygdalohippocampal area (AHi, Fig. $2 \mathrm{~h}$ ). It is noteworthy that the pallial telencephalon is one of the few brain sites where the pSTAT5-ir pattern of our pregnant and lactating females showed a certain degree of variability. This variability affects mainly the piriform cortex (Pir), where pSTAT5 immunoreactivity was observed in just one animal (a bromocriptine-treated 
pregnant female; see Fig. 2d) and the cortical amygdala. Here, inter-individual variability did not correlate with the physiological status of our experimental groups, as it was observed across untreated pregnant, and bromocriptine-treated pregnant and lactating females. Hence, 7 out of 18 animals displayed pSTAT5-ir in the ACo, 6 in the LOT, 7 in the BLV, and only 3 animals in the BMP and AHi. In all these cases, immunoreactive cell bodies were sparse and faintly stained. In contrast, the BMA showed pSTAT5-ir in all the animals.

In the subpallial telencephalon, pSTAT5-ir was abundant in the septum, the extended amygdala (EA), and the anterodorsal amygdaloid area (AAD). Within the EA, both the central and medial EA displayed immunolabelling. Thus, pSTAT5 was present in the medial and central nuclei of the amygdala, in different subnuclei of the bed nucleus of the stria terminalis (BST), as well as in the sublenticular substantia innominata (SI, Fig. $3 \mathrm{~b}$ ) and the interstitial nucleus of the posterior limb of the anterior commissure (IPAC, Fig. $2 \mathrm{c}-\mathrm{e}$ ).

Fig. 3

Representative examples of pSTAT5 immunoreactivity in the telencephalon, midbrain, and brainstem of late-pregnant and lactating female mice. Photomicrographs showing the pSTAT5-ir pattern in representative brain regions of pregnant and lactating mice. Sections include the lateral septum and anterior BST (a); the central and medial anterior (b) and posterior (c) amygdala; the periaqueductal grey (d); the parabrachial complex (e), and the nucleus of the solitary tract and surrounding structures (f). Sections $\mathbf{b}$ and $\mathbf{f}$ correspond to a postconception day 14 pregnant female (specimen M1435), sections a and d to a bromocriptine-treated, day 14 pregnant female (specimen M1392), and sections c and $\mathbf{f}$ to a post,partum day 6 lactating dam (specimen M13111). The approximate distance to bregma is indicated in each section. Scale bars $250 \mu \mathrm{m}$ 


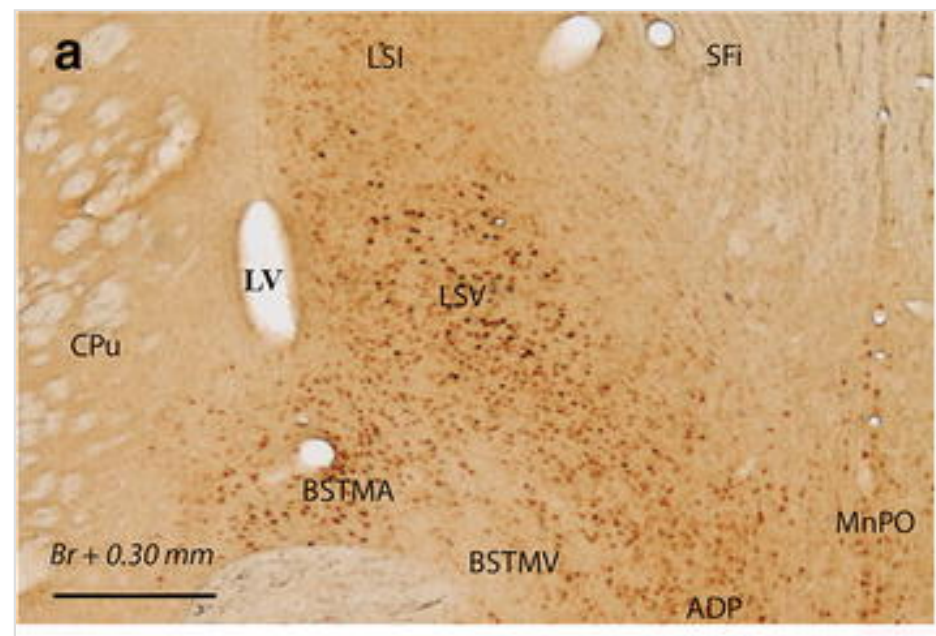

\section{b} CEMAD

$\mathrm{CeC}$

BMA
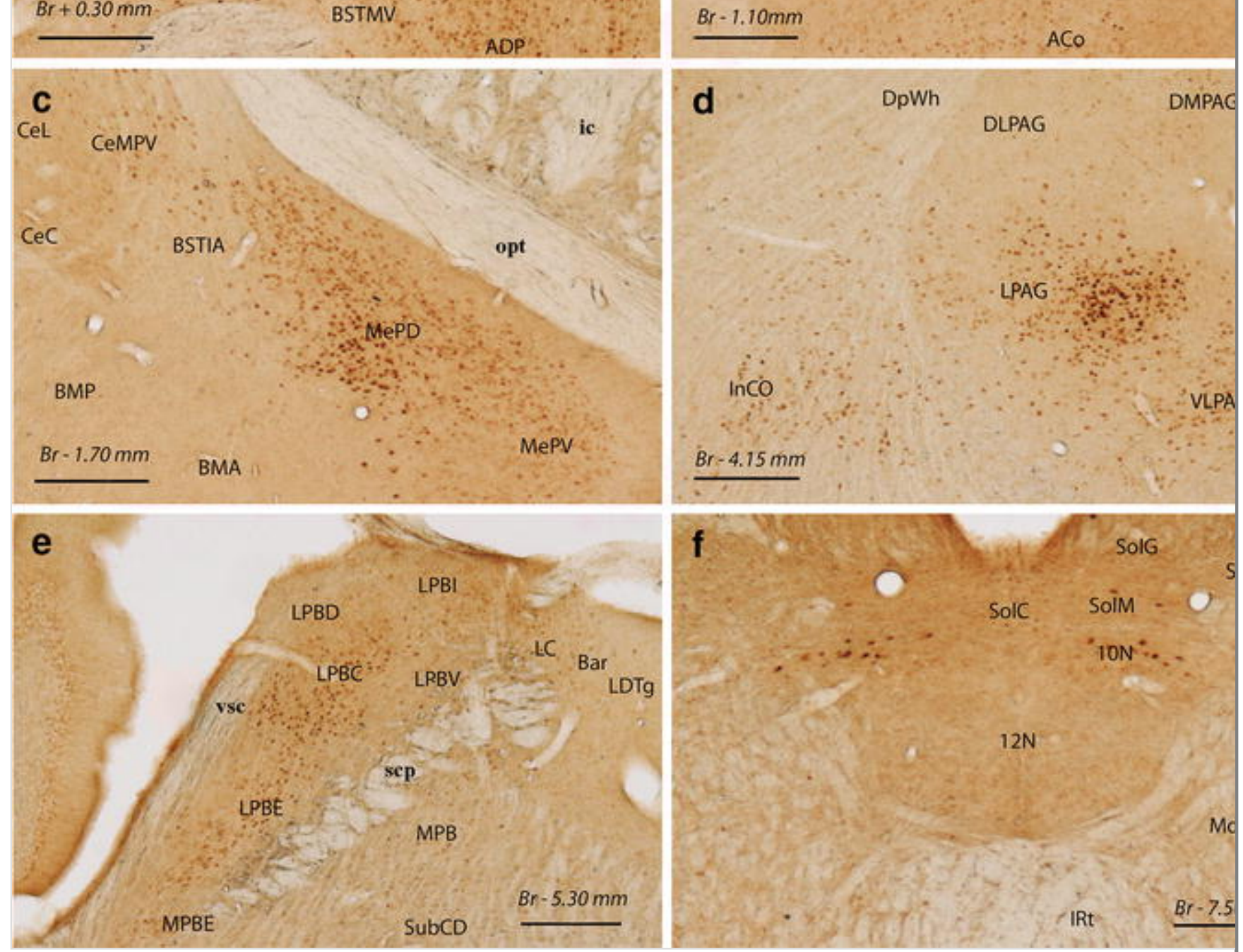

The medial extended amygdala (MEA) displayed pSTAT5-ir in all of its nuclei. Within the amygdala proper, pSTAT5-ir was moderately dense in the anterior (MeA, Fig. 3 b), post-eroventral (MePV, Fig. $3 \mathrm{c}$ ), and, especially, the posterodorsal (MePD, Fig. 3c) nuclei of the medial amygdala, as well as in the intraamygdaloid division of the BST (BSTIA, Fig. $3 \mathrm{c}$ ). In addition, a high density of immunolabelled cells was observed in the medial (BSTMPM), lateral (BSTMPL), and (with a lower density) intermediate (BSTMPI) nuclei of the post-eromedial BST (Figs. 1 a-c, 2d).

As for the central extended amygdala (CEA), within the central amygdaloid nucleus, pSTAT5-ir was almost restricted to the medial division of the $\mathrm{Ce}(\mathrm{CeM}$, 
Fig. $3 \mathrm{~b}, \mathrm{c})$, although a small number of cells also appeared in the lateral $\mathrm{Ce}$ (CeL, Fig. $3 \mathrm{c}$ ). Within the BST, most of the nuclei in the anterior and lateral BST displayed abundant labelling: the medial anterior (BSTMA, Fig. $3 \mathrm{a}$ ), medial ventral (BSTMV, Fig. 4a), and lateral nuclei of the BST (ventral BSTL, posterior BSTL, dorsal BSTL, Fig. 2c, d), with the exception of the juxtacapsular nucleus (BSTLJ, Fig. 2c).

Fig. 4

Representative examples of pSTAT5 immunoreactivity in the diencephalon of latepregnant and lactating female mice. Photomicrographs showing the pSTAT5-ir pattern in representative brain regions of pregnant and lactating mice. Sections include the preoptic hypothalamus (a), the paraventricular and supraoptic nuclei in the anterior hypothalamus (b), the arcuate and ventromedial nuclei in the tuberal hypothalamus (c), the habenular complex (d), the premammillary hypothalamic region (e), and the posterior thalamic region (f). Sections $\mathbf{a}$ and $\mathbf{b}$ correspond to a post-conception day 14-pregnant female (specimen M1435), sections $\mathbf{c}$ and $\mathbf{d}$ to a bromocriptine-treated, day 15-pregnant female (specimen M1392), and sections e and $\mathbf{f}$ to a post-partum day 6 lactating dam (specimen M13111). The approximate distance to bregma is indicated in each section. Scale bars $250 \mu \mathrm{m}$ 

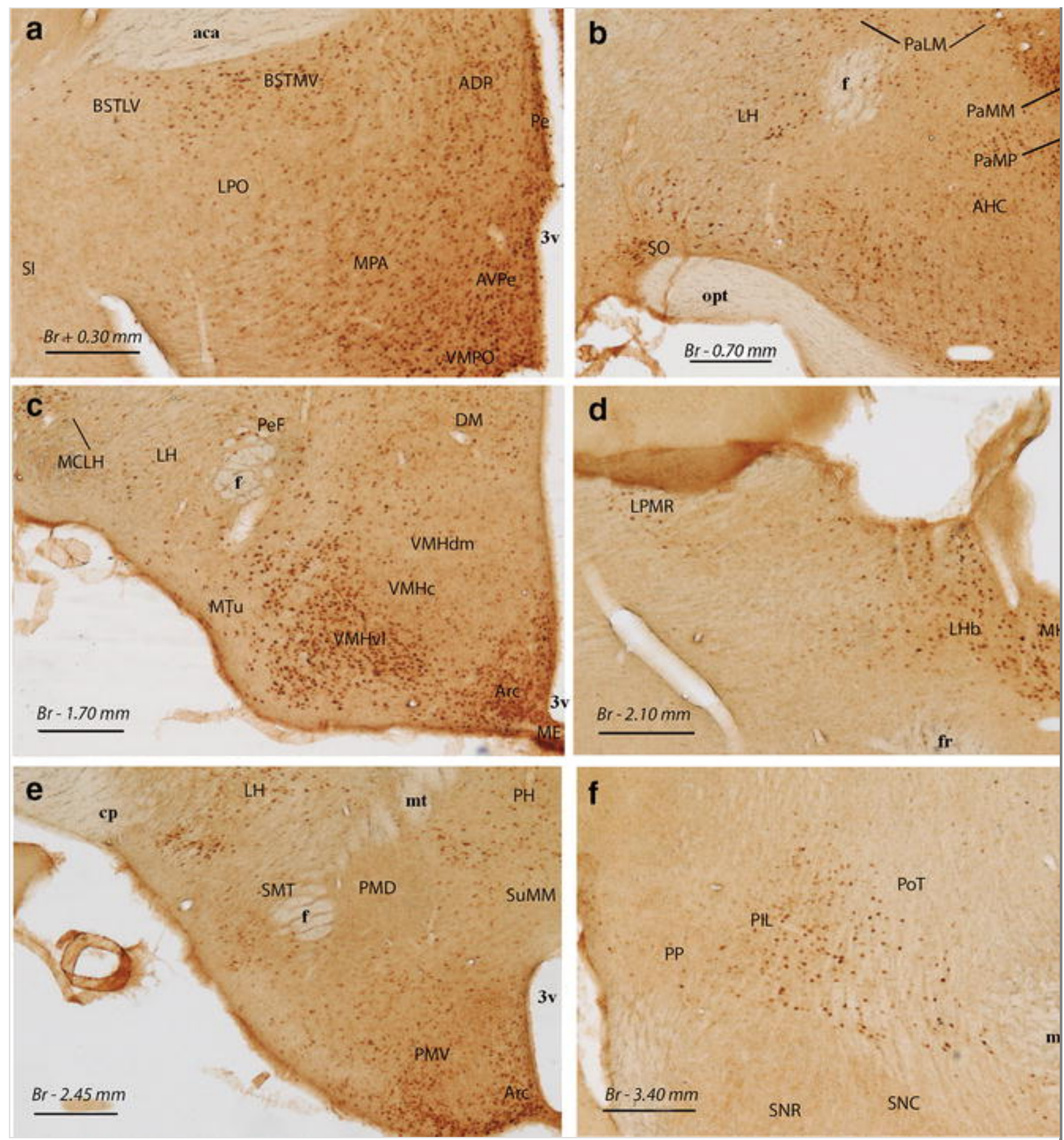

Within the basal cerebral hemispheres, we observed pSTAT5-ir cells in a portion of the ventral striato-pallidum located between the diagonal band nucleus and the shell of the nucleus accumbens, named as medioventral striato-pallidum (OteroGarcia et al. 2014), because of its relationship with structures of the ventral striatum and pallidum, or ventral septal area (Rood and De Vries 2011), given its apparent continuity with the lateral septum (see Fig. 2b).

In the septum, we found pSTAT5-ir within the different nuclei of its lateral division (LSV, LSI, and LSD, Figs. 2b, 3 a) and in the septo-hypothalamic nucleus (SHy, not shown). Some structures in the medial and posterior septal regions contained pSTAT5-ir, too, such as the septohippocampal (SHi, Fig. 2b) 
and septofimbrial (SFi, Fig. 3 a) nuclei and the bed nucleus of the anterior commissure (BAC, Fig. 2d). Finally, pSTAT5 immunostaining was found in the subfornical organ (SFO, Fig. 2d).

\section{Diencephalon}

Immunoreactivity for pSTAT5 was abundant in many structures of the diencephalon of late-pregnant and lactating females, including hypothalamic, thalamic, and pretectal nuclei.

Apparent pSTAT5-ir was observed in the four major rostro-caudal divisions of the hypothalamus, as well as in the three medio-lateral compartments (periventricular, medial, and lateral). Figure 4 illustrates the appearance of the pSTAT5-ir pattern in representative examples of the aforementioned sites.

The preoptic region (Figs. 1, 4a) displayed high levels of pSTAT5 expression in the structures surrounding the third ventricle, namely in the anteroventral periventricular (AVPe), ventromedial preoptic (VMPO), and median preoptic $(\mathrm{MnPO})$ nuclei, as well as the periventricular nucleus $(\mathrm{Pe})$ and the vascular organ of the lamina terminalis (VOLT, not shown). The medial region of the preoptic hypothalamus displayed widespread pSTAT-ir, too. Labelling was present in the medial preoptic area (MPA) and in every division of the medial preoptic nucleus (MPO, Fig. $1 \mathrm{a}-\mathrm{c}$ ), as well as in dorsal preoptic structures, such as the nucleus of the anterior commissure (AC, Fig. $1 \mathrm{a}-\mathrm{c}$ ) or the anterodorsal preoptic nucleus (ADP). Finally, within the lateral compartment, the bed nucleus of the stria medullaris (SM, not shown), the lateral preoptic (LPO), and the ventrolateral preoptic (VLPO, not shown) areas displayed scattered pSTAT5 positive cells.

In the anterior hypothalamus (Figs. $2 \mathrm{e}-\mathrm{f}, 4 \mathrm{~b}$ ), the most outstanding pSTAT5-ir cell groups corresponded to the paraventricular $(\mathrm{Pa})$ and supraoptic nuclei ( $\mathrm{SO}$, including its retrochiasmatic region, SOR), with a high density of immunostained neurons. Within the Pa complex, labelled cells were observed not only in its magnocellular division (medial, PaMM; lateral, PaLM), but also in the remaining subnuclei, PaAP, PaDC (not shown), PaV (Fig. 2e), and PaPo (Fig. 2f). Other nuclei containing magnocellular neurosecretory neurons, such as the suprachiasmatic nucleus, were remarkably devoid of pSTAT5 immunostaining. Additional sparser populations of pSTAT5-positive cells were present in the subparaventricular nucleus ( $\mathrm{SPa}$ ) and Pe within the juxtaventricular compartment, through the anterior hypothalamic area (AHA, AHC, and AHP, Fig. 2 e, f), lateroanterior (LA, Fig. 2e), and circular (Cir, Fig. 2e) nuclei of the medial compartment, and in the lateral hypothalamic area $(\mathrm{LH})$. 
In the tuberal hypothalamus (Fig. $4 \mathrm{c}$ ), the highest density of pSTAT5-ir cells was observed in the arcuate nucleus (Arc), presumably, including the TIDA neurons (Yip et al. 2012). Remarkably, a few cells extended from this cluster into the median eminence (ME, Fig. $2 \mathrm{~g}$ ). In the medial compartment, dense groups of labelled cells were observed in the dorsomedial (DM) and the ventromedial nuclei (VMH). Within the VMH, the highest levels of pSTAT5-ir corresponded to the ventrolateral subdivision (VMHvl), while the central (VMHc) and dorsomedial VMH subnuclei (VMHdm) showed only scattered and faintly stained cells (Fig. 4c). Interestingly, the cluster of immunostained nuclei in the VMHvl is not confined to the boundaries of the VMH, but extends further laterally into the ventromedial aspect of the lateral hypothalamic area (see Fig. $4 \mathrm{c}$ ). The rest of the LH displayed a moderate-to-low density and intensity of immunolabelling. Other centres showing pSTAT5-ir in the tuberal hypothalamus include the subincertal (SubI, Fig. 2g), perifornical (PeF), and the medial tuberal (MTu) nuclei, as well as the magnocellular (MCLH) and parasubthalamic (PSTh, Fig. 4 e) divisions of the lateral hypothalamic area.

In the premammillary hypothalamus (Fig. $4 \mathrm{e}$ ), a dense population of pSTAT5-ir cells was observed in the ventral premammillary nucleus (PMV). In the dorsal premammillary nucleus (PMD), pSTAT5-ir was absent in lactating females and present only in half of the animals in the pregnant groups. Finally, the medial supramammillary nucleus (SuMM), the submammillothalamic nucleus (SMT), and the posterior hypothalamic area (PH, Figs. $2 \mathrm{~h}, 4 \mathrm{e})$ displayed scattered immunostaining.

Immunoreactivity for pSTAT5 was observed in several of the nuclear groups of the thalamus. Labelling was present in the ventral thalamus, in some nuclei of the midline dorsal thalamus and habenular complex, in several visual and auditory thalamic nuclei, and in the posterior-intralaminar thalamic complex.

In the prethalamus, labelling was present in the zona incerta (ZI, Fig. $2 \mathrm{f}-\mathrm{h}$ ) and the reticular thalamic nucleus (Rt, Fig. $2 \mathrm{e}, \mathrm{f}$ ). The Rt showed a variable staining frequency in our sample: labelling was only observed in approximately $50 \%$ of the late-pregnant females examined, in which staining was relatively weak and sparse as compared to other regions of the brain.

In the midline thalamus, a few cells were visible in the ventral reuniens nucleus (VRe, Fig. 2g) and a larger population of pSTAT5 immunoreactive cells was seen in the paraventricular nucleus (PV, Fig. 2 g). Next to the PV, some pSTAT5ir cells were found within the caudalmost levels of the habenular complex, which represents a notable exception to the generally stable pattern of pSTAT5-ir. First, staining in the medial $(\mathrm{MHb})$ and lateral $(\mathrm{LHb})$ habenula was not present in 
lactating females (where the habenular complex is completely blank), but only in some pregnant females, either untreated or treated with bromocriptine. In these animals, labelling in the habenula was variable and heterogenous: the $\mathrm{MHb}$ appeared only occasionally stained, whereas the LHb was labelled in $50 \%$ of the animals (see Fig. $4 \mathrm{~d}$ for a case where both subdivisions are positively labelled and Fig. $2 \mathrm{~g}$ for a different case).

Several nuclei of the visual thalamus displayed pSTAT5 immunolabelling. Within the lateral geniculate complex, labelled cells were seen especially in the intergeniculate leaflet (IGL, Fig. $2 \mathrm{~h}$ ), but also in the ventral geniculate nucleus (VLG), almost restricted to its parvocellular subdivision (VLGPC, Fig. 2h). Furthermore, it is noteworthy that a small number of immunolabelled cells was found in an unnamed location near to the dorsal division of the medial geniculate nucleus (Fig. 2i).

However, the largest populations of immunostained cells appeared in several nuclei of the posterior thalamus, including the lateral posterior (LP, Fig. 2h), parafascicular (PF), and subparafascicular (SPF, with its parvocellular part, SPFPC) nuclei (Fig. 2h), the retroethmoid nucleus (REth, not shown), the prerubral field (PR, Fig. 2h), the posterior intralaminar complex (PIL), and the triangular part of the posterior thalamus (PoT). These last two nuclei (PIL and PoT) form a dense and remarkable stained cell population in the posterior thalamus, in which a few cells seem to be displaced caudoventrally into an unnamed area just dorsal to the substantia nigra pars compacta (see Figs. 2i, 4f).

The pretectum comprises the limit between the thalamus and the midbrain (Martinez-Ferre and Martinez 2012). There, structures immunolabelled for pSTAT5 included the posterior (PPT) and olivary (OPT) pretectal nuclei (both immunostained in half of the pregnant and lactating animals, see Fig. $2 \mathrm{~h}$ ), the precommissural nucleus ( $\mathrm{PrC}$, not shown), flanking the posterior commissure, and the nucleus of the posterior commissure (PCom), including its magnocellular division (MCPC). Exceptionally, the subcommissural organ (SCO, Fig. 2h), unlike the rest of the reviewed circumventricular organs, appeared devoid of pSTAT5 immunoreactivity.

\section{Midbrain and brainstem}

In the midbrain, lactotroph-derived signal transduction was observed mainly in the periaqueductal grey (PAG), but also in the adjoining lateral tegmentum and, further dorsally, in the superior colliculus (see Fig. $3 \mathrm{~d}$ ).

The PAG displayed a heterogeneous pSTAT5-ir. The most prominent labelling corresponded to the lateral PAG (LPAG). Second, the ventrolateral (VLPAG) and 
dorsomedial (DMPAG) columns showed moderate immunoreactive cells. Finally, the dorsolateral PAG (DLPAG) displayed sparse immunoreactive cells. The Edinger-Westphal nucleus (EW, Fig. 2i), embedded between the VLPAG and the oculomotor nuclei, was also positively labelled for pSTAT5, as were the nucleus of Darkschewitsch (Dk) and the interstitial nucleus of Cajal (InC) (both located lateral to the anterior periaqueductal grey, PAG, see Fig. 2i).

Dorsal to the PAG, some immunostained cells appeared in the deep (DpG and DpWh, the latter seen in Fig. 3d) and intermediate (InG and InWh, Fig. 2 i) layers of the superior colliculus and in the intercollicular nucleus (InCO, Fig. 4 d). Within the tegmentum, pSTAT5-it was present in the deep mesencephalic area (DpMe, Fig. 3d), the nucleus of the brachium of the inferior colliculus (BIC, Fig. 2j), and the interpeduncular nucleus (IP, Fig. 2i).

In the rhombencephalon, pSTAT5-ir was remarkably absent in the cortex and deep nuclei of the cerebellum. By contrast, the pons showed a complex pattern of pSTAT5-ir. In the pontine tegmental region, pSTAT5-immunoreactive cells appeared in the pedunculopontine tegmental nucleus (PPTg, Fig. $2 \mathrm{j}$ ), the dorsomedial tegmental nucleus (DMTg, Fig. $2 \mathrm{k}$ ), and the laterodorsal tegmental nucleus (LDTg, Fig. 3 e), and in Barrington's nucleus (Bar, Fig. $3 \mathrm{e}$ ), which displayed a small number of weakly stained cells. Scattered immunostained neurons appeared in the oral (PnO, Fig. 2j) and caudal parts (PnC, Fig. 2k) of the pontine reticular formation. Furthermore, the locus coeruleus (LC) and the parabrachial complex (PB) displayed relatively abundant immunostaining (Fig. $3 \mathrm{e}$ ). Within the parabrachial complex, the highest labelling density was seen in the central (LPBC) and external (LPBE) parts of the lateral parabrachial nucleus (LPB), with the rest of this nucleus (LPB) and the medial parabrachial nucleus (MPB) containing a lower density of immunoreactive cells.

The raphe nuclear complex displayed ample pSTAT5-ir. At rostral levels, the median (MnR), dorsal (DR) and the caudal linear (CLi) nuclei of the raphe were positively stained for pSTAT5 (Fig. 2j). Caudally, pSTAT5-ir was still observed in the DR, in the pontine nucleus (PnR, Fig. $2 \mathrm{k}$ ), and in the raphe magnus (RMg, Fig. 2k). Finally, the raphe obscurus (ROb) in the caudalmost region of the hindbrain (Fig. 21) also displayed pSTAT5 immunostained cells.

As for the reticular nuclei, pSTAT5-ir appears in the subcoerulear nucleus, and ventral and dorsal divisions (SubCV and SubCD, respectively, the latter seen in Fig. 3e). More caudally, sparse immunoreactive cells appear in the dorsal and ventral medullary (MdD, MdV, the latter seen in Fig. $3 \mathrm{f}$ ) and intermediate (IRt) reticular nuclei, in the ambiguus nucleus (Amb) and, laterally, along the spinal trigeminal nucleus (SP5), in close contact with the spinal trigeminal tract (see 
Fig. 21).

Finally, like other circumventricular sites, the area postrema (AP) and the adjoining nuclei, i.e., nucleus of the solitary tract (Sol) and dorsal motor nucleus of the vagus $(10 \mathrm{~N})$, showed prominent pSTAT5-ir, as seen in Fig. $3 \mathrm{f}$.

\section{Immunoreactivity for $\mathrm{pSTAT}_{5}$ in the brain of virgin female mice}

As a rule, virgin females showed less labelling than pregnant and lactating ones. In fact, the patterns of pSTAT5-ir observed in virgin females were always a fraction of the one just described for pregnant/lactating females, so that no brain centre was stained for pSTAT5 in virgins, but not in pregnant/lactating females. However, and in contrast to pregnant/lactating mice, virgin females showed a substantial inter-individual variability in the presence of pSTAT5 immunolabelled cells in several brain regions. We have illustrated this by establishing a frequency score for each brain site, according to the proportion of virgin females of our sample $(n=7)$ showing pSTAT5 immunoreactive cells in that site. This score is color-coded in Fig. 2 and comprises four categories: (a) no labelling in any virgin female (clear background), (b) low frequency of pSTAT5-ir (1-2 animals, blue background); (c) intermediate frequency of pSTAT5-ir (3-5 animals, pink background); and (d) high frequency of pSTAT5-ir (6-7 animals; orange). In addition, we provide some supplementary examples of the qualitative differences in pSTAT5-ir that our virgin female sample displays in several representative brain sites (supplemental material, OR2).

According to our results, only two nuclei in the whole brain showed labelling consistently in every virgin female of our sample: the Arc (Fig. OR2b) and the MePD (Fig. OR2a). Three more brain centres displayed pSTAT5-ir in most animals, namely the LSV (Fig. OR2a), the ventrolateral VMH (Fig. OR2b), and the rostral and lateral PAG (Fig. OR2a). By contrast, the majority of the analysed nuclei showed labelling in 3-5 of the 7 animals. Finally, some brain regions were totally devoid of pSTAT5-ir in the whole sample of virgin females. These include portions of the allocortex (DTT, EnD), and specific nuclei of the amygdala (AAD-AAV, LOT, and BLV), basal telencephalon (BAC), thalamus or pretectum (Rt, LHb, PPT, LP, and PF), and dorsal and pontine tegmental nuclei (Bar, LDTg, DMTg, and RMg).

\section{Quantitative analysis of pSTAT5-ir in the brain of virgin, pregnant, and lactating females}

We assessed the density of pSTAT5 immunoreactive cell nuclei in 13 brain regions chosen according to their relevance in the context of maternal behaviour 
regulation. These regions comprise several nodes of the sociosexual brain: the LSV, the MePD, BSTMPM, AC/ADP, MPO, VMHvl, and adjoining tuberal region (hypothalamic aggression locus), and VLPAG (Newman 1999). We also included the magnocellular neurosecretory nuclei ( $\mathrm{Pa}$ and $\mathrm{SO}$ ) and other sites that are also involved in the regulation of maternal behaviours (CeM, AVPe, and PIL; Bosch and Neumann 2010; Scott et al. 2015) or related aspects of motherhood (Cservenák et al. 2013), despite not being usually considered as part of the sociosexual brain network. Finally, we also analysed the Arc, given its role in the feedback control of hypophyseal PRL release (Ben-Jonathan and Hnasko 2001a; Sapsford et al. 2012). The exact frames applied for each of these nuclei are depicted in Fig. 5.

\section{Fig. 5}

Anatomical location of pSTAT5-ir counting frames. Semi-schematic camera-lucida drawings of selected coronal sections featuring the counting frames (red) chosen for pSTAT5-ir quantification: a LSV; b AVPe; $\mathbf{c}$ BSTMPM; d AC/ADP; e MPO; f SO; $\mathbf{g ~ P a}$; h CeM; i VMHvl and ventrolateral adjoining area; j Arc; k MePD; I PIL and adjoining posterior thalamic region; and $\mathbf{m}$ VLPAG. The green box in the MPO represents the modified frame employed in ovariectomized virgin female mice, restricted to the medial half of the nucleus. Approximate distance to Bregma enclosed for each section. Scale bars $500 \mu \mathrm{m}$ 
e.Proofing
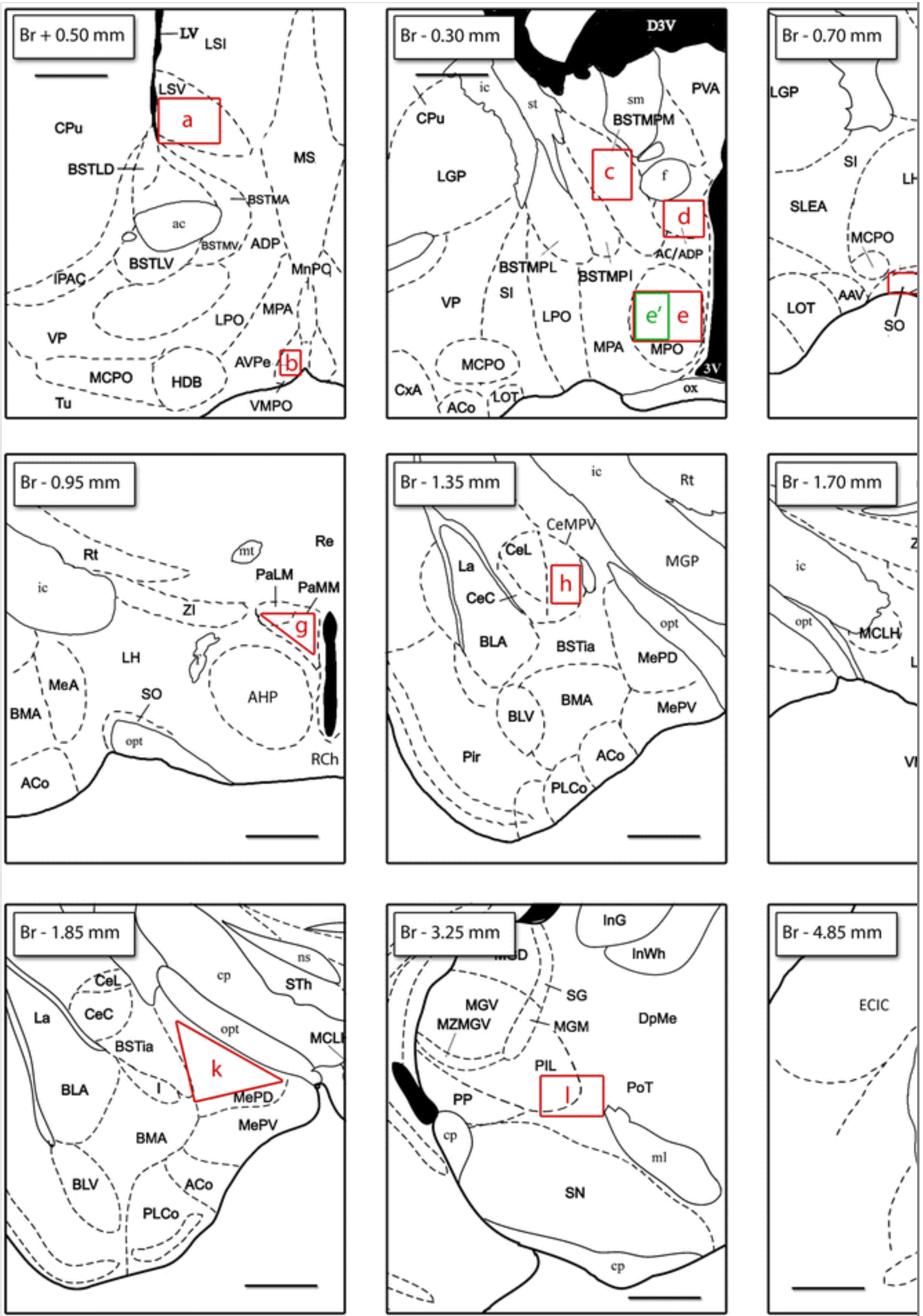

First, we compared pSTAT5-ir density between bromocriptine-treated pregnant females and non-treated pregnant females, to rule out any possible effect of 
impairment of hypophyseal PRL release on the levels of central lactogenic signalling. To do so, we performed a $t$ test for independent samples for each of the analysed nuclei. $t$ tests revealed no significant differences between treated and non-treated pregnant females in any case. $p$ values were always equal or higher than 0.2 , with the single exception of the CeM, where a trend towards significance was found ( $p=0.064)$. Therefore, our results confirm no significant effect of bromocriptine treatment in pSTAT5-ir density in late-pregnant females. Consequently, we focus our following analysis on virgins, non-treated pregnant females, and lactating dams.

To explore the variation of pSTAT5-ir density between virgin, pregnant, and lactating groups, we performed a separate one-way ANOVA on each of the sampled nuclei. The results of this analysis are summarized in Fig. 6 and reveal statistically significant inter-group differences in pSTAT5-ir density in most of these brain regions. The main effects revealed by ANOVA were further explored using post-hoc Bonferroni multiple comparisons. The results of this post-hoc analysis indicate that virgins generally showed significantly less pSTAT5-ir density than one or more of the remaining groups.

\section{Fig. 6}

Quantification of pSTAT5-ir density in selected brain regions of virgin, pregnant, and lactating female mice. Assessment of pSTAT5-ir density (pSTAT5-positive cell nuclei $/ \mathrm{mm}^{2}$ ) in 13 brain regions of relevance in the context of maternal behaviours (Newman 1999; Gammie 2005). Bar histograms show mean interhemisferic pSTAT5-ir density \pm SEM in virgin female mice ( $n=7$; white); PD $14-18$ latepregnant mice $(n=5$; grey) and PPD6 lactating dams $(n=6$; black $)$. Statistical analysis (one-way ANOVA with Bonferroni post-hoc comparisons) was applied independently to each brain region. ${ }^{*} p \leq 0.05 ;{ }^{* *} p \leq 0.01 ;{ }^{* * *} p \leq 0.001$ 

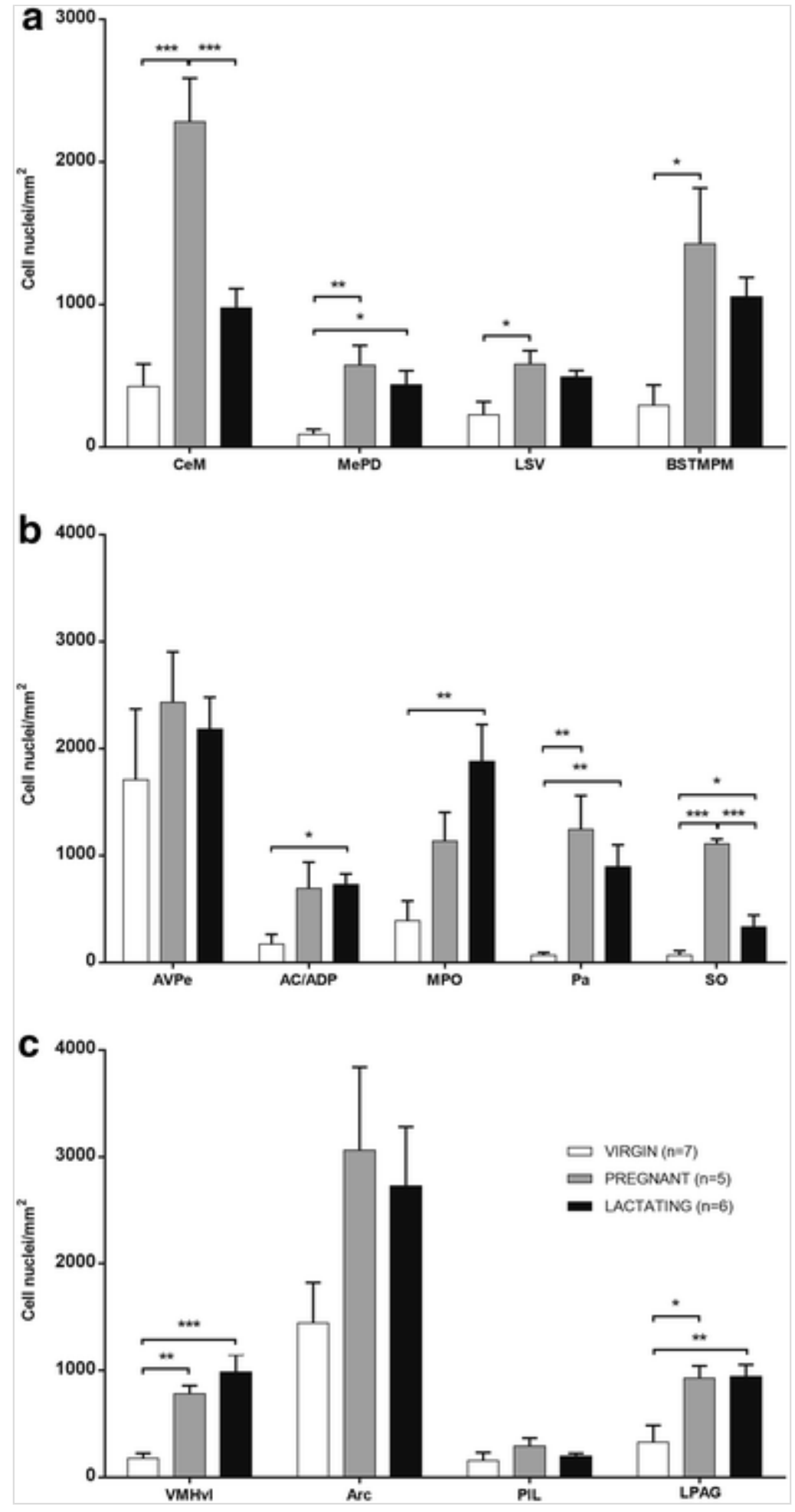

The ANOVA of the density of pSTAT5-ir revealed significant differences between groups within the nuclei of the extended amygdala, namely the CeM $\left(F_{2,15}=22.071, p<0.001\right), \operatorname{MePD}\left(F_{2,15}=8.016, p=0.004\right)$, and BSTMPM $\left(F_{2,15}=6.243, p=0.012\right)$, and in the septal region $\left(\mathrm{LSV} ; F_{2,15}=5.219\right.$, $p=0.019$ ) (Fig. 6a). Post-hoc comparisons indicate that in the CeM, pSTAT5-ir density was significantly higher in pregnant than in virgin females $(p<0.001)$ or lactating dams $(p=0.001)$. On the other hand, the density of immunoreactive cells in lactating dams was similar to that of virgins $(p=0.174)$. Concerning the MePD, labelling density was significantly higher in pregnant females than in virgin females $(p=0.005)$ and in lactating dams compared to virgin females 
$(p=0.037)$, whereas both pregnant females and lactating dams displayed very similar labelling density $(p=0.949)$. Regarding the BSTMPM, labelling density in pregnant females was significantly higher than in virgins $(p=0.012)$, but similar to that of lactating dams $(p=0.836)$. On the other hand, although the average density of pSTAT5 immunoreactive cells was higher in dams than in virgin females, this difference did not reach significance $(p=0.091)$. Finally, the LSV showed a significant increase in pSTAT5-ir density in the brain of pregnant females as compared to that of virgin females $(p=0.027)$, but pregnant and lactating females showed identical levels $(p=1)$, whereas the apparent differences between virgin and lactating groups were not significant $(p=0.093)$.

In the preoptic hypothalamus (Fig. 6b), significant differences in labelling density were observed in the $\operatorname{AC} / \operatorname{ADP}\left(F_{2,15}=5.141, p=0.020\right)$ and MPO $\left(F_{2,15}=8.345, p=0.004\right)$, but not in the AVPe $\left(F_{2,15}=0.522 ; p=0.605\right)$. In the AC/ADP, post-hoc comparisons indicate that virgin females had significantly lower pSTAT5-ir density than lactating dams $(p=0.035)$, as well as a trend to lower pSTAT5-ir than pregnant females $(p=0.067)$. By contrast, pregnant and lactating dams showed identical density of immunolabelled cells $(p=1)$. For the MPO, one of the key nodes of the maternal brain, lactating dams showed significantly higher pSTAT5-ir density than virgin females $(p=0.003)$, whereas the rest of the comparisons did not reach significance $(p \approx 0.25$ for both comparisons).

In the anterior hypothalamus (Fig. $6 \mathrm{~b})$, the paraventricular $\left(F_{2,15}=10.099\right.$, $p=0.002)$ and supraoptic nuclei $\left(F_{2,15}=52.827, p<0.001\right)$ showed significant inter-group differences. In the $\mathrm{Pa}$, both pregnant and lactating groups showed similar densities of pSTAT5-ir $(p=0.715)$, which are significantly higher than in virgin females ( $p=0.002$ and $p=0.02$, respectively). In the SO, pregnant females displayed significantly higher pSTAT5-ir density than virgin females and lactating dams $(p<0.001)$, whereas lactating dams also displayed significantly higher levels than virgin females $(p=0.049)$.

In the tuberal hypothalamus (Fig. 6c), the VMHvl and laterally adjoining area showed significant inter-group differences in pSTAT5-ir density $\left(F_{2,15}=16.673\right.$, $p<0.001)$. Thus, virgin females displayed significantly lower labelling density than pregnant females $(p=0.004)$ and lactating dams $(p<0.001)$, whereas pregnant females and lactating dams showed similar labelling density $(p=0.687)$. Conversely, the ANOVA revealed no significant differences in labelling density in the sampled area of the arcuate nucleus $\left(F_{2,15}=2.502\right.$; $p=0.115)$.

Similarly, no group effect was observed in the PIL within the posterior thalamus 
$\left(F_{3,22}=1.194, p=0.335\right.$; see Fig. $\left.6 \mathrm{c}\right)$. Finally, in the periaqueductal grey (VLPAG), the ANOVA revealed a significant group effect $\left(F_{2,15}=7.475\right.$, $p=0.006)$. The post-hoc analysis indicates that pregnant and lactating groups showed significantly higher labelling density than virgins $(p=0.029$ and $p=0.012$, respectively) and identical between each other $(p=1)$.

Summarizing, this quantitative analysis reveals that in most of the analysed brain regions, pSTAT5-ir density (as a measure of lactogenic activity) increased significantly during pregnancy and/or lactation. Furthermore, we found no statistically significant effect of bromocriptine treatment in pSTAT5-ir density in the sampled structures. Finally, levels of pSTAT5-ir in the analysed structures do not differ between late-pregnancy and lactation, with the exception of the CeM and the $\mathrm{SO}$, where labelling density decreases from late-pregnancy to lactation.

\section{Quantitative analysis of central pSTAT5-ir density in steroid- treated, ovariectomized virgin female mice}

To explore the putative role of gonadal steroids in modulating central PRL signalling, we conducted a hormonal substitution experiment: ovariectomized virgin female mice were treated with vehicle (OO), estradiol (EO), or estradiol plus progesterone (EP) (see "Materials and methods"). We assessed the density of pSTAT5 immunoreactive cells in these samples in 12 out of the 13 counting frames designed for the former analysis (see Fig. 5). The SO was left out of this analysis, since it presented negligible levels of pSTAT5-ir in all of the experimental groups. Aside from this exception, the counting frame for the MPO was modified, since pSTAT-ir was clearly restricted to the medial half of the nucleus. Accordingly, the frame was limited to this area (Fig. 5).

Separate non-parametric one-way Kruskal-Wallis ANOVA tests revealed significant differences in pSTAT5-ir density between OO, EO, and EP groups in 5 out of the 13 analysed nuclei (Fig. 7). Discrete inter-group differences were further explored with Dunnett's post-hoc method for multiple comparisons, which revealed significant effects generally consisting of an important increase in pSTAT5-ir density affecting one or both groups treated with estradiol (EO and EP) as compared to the vehicle group (OO). This analysis indicated significant differences in pSTAT5-ir density in every studied nucleus of the telencephalon except for the CeM $(p=0.246)$. In the MePD $\left(\chi^{2}(2)=9.748 ; p=0.001\right)$, the EP group displayed significantly higher pSTAT5-ir density than the OO group $(p=0.007)$, whereas differences between the EO and OO group did not reach significance $(p=0.07)$. In the BST region, we found a trend towards inter-group differences in the BSTMPM $\left(\chi^{2}(2)=5.558 ; p=0.062\right)$. In the septal region, we found a significant effect in the $\operatorname{LSV}\left(\chi^{2}(2)=11.263 ; p=0.004\right)$, where 
pSTAT5-ir density of the EO and EP groups are again significantly higher than the OO group ( $p=0.004$ and $p=0.038$, respectively).

\section{Fig. 7}

Quantification of pSTAT5-ir density in selected brain regions of ovariectomized, estrogen-treated virgin female mice. Assessment of pSTAT5-ir density (pSTAT5positive cell nuclei $/ \mathrm{mm}^{2}$ ) in 12 brain regions involved in the management of social and reproductive behaviours and in the control of PRL release (Newman 1999; Ben-Jonathan and Hnasko 2001b; Gammie 2005). Bar histograms show mean interhemisferic pSTAT5-ir density \pm SEM in ovariectomized virgin female mice treated with: vehicle (group OO; black; $n=6$ ), estradiol (group EO; white; $n=6$ ) or estradiol + progesterone (group EP; grey; $n=6$ ). Non-parametric KruskalWallis ANOVA with Dunnett's post-hoc pairwise comparisons was applied separately to each brain region. ${ }^{*} p \leq 0.05 ; * * p \leq 0.01$ 

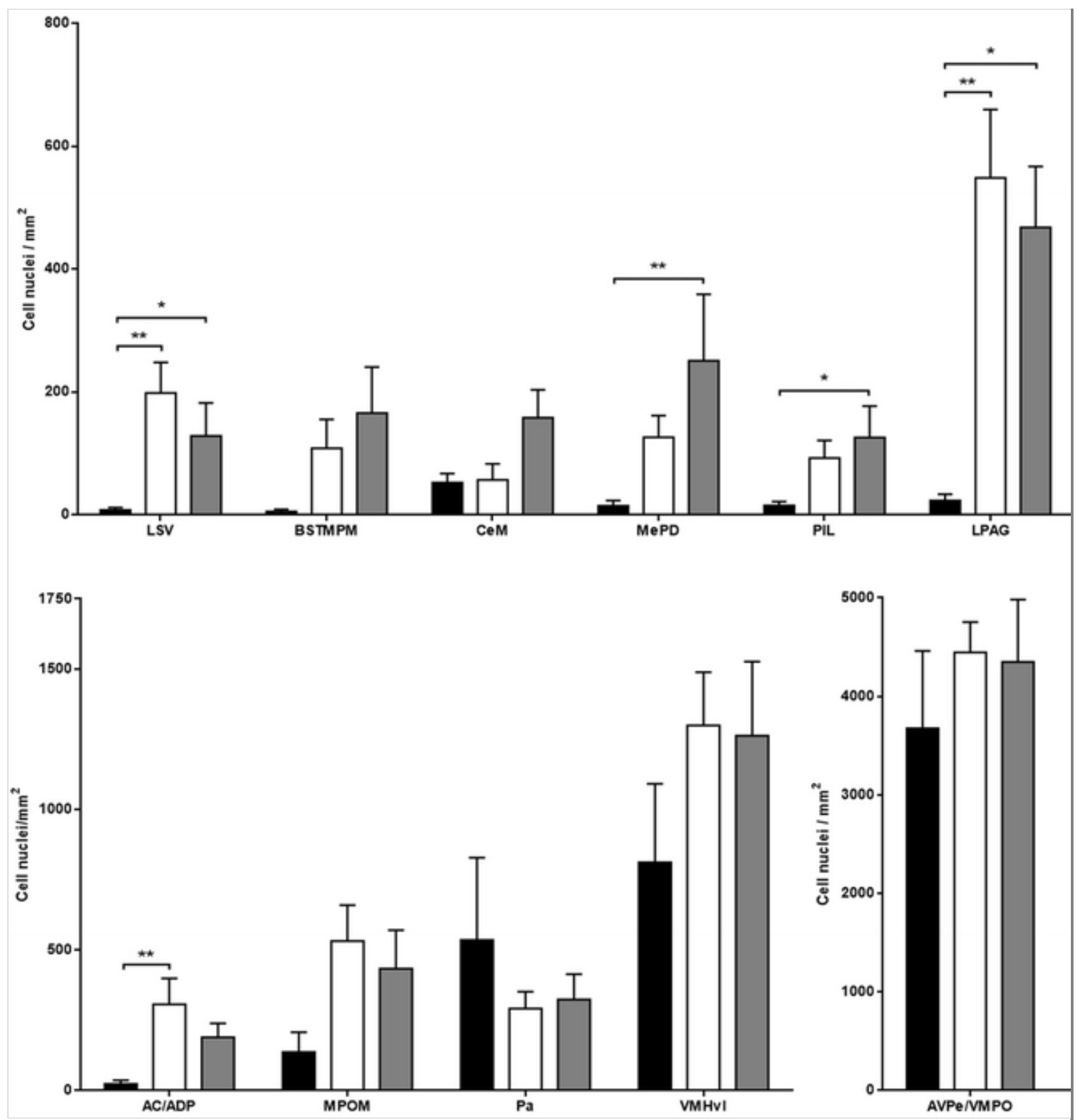

In the preoptic hypothalamus, a significant effect of the treatment was observed in the AC/ADP $\left(\chi^{2}(2)=9.182 ; p=0.010\right)$, but not in the AVPe/VMPO $(p=0.806)$ and MPOM $(p=0.08)$. In the AC/ADP, post-hoc analysis determined that the EO group had significantly higher pSTAT5-ir density than the control $(p=0.01)$, whereas differences between the control and EP group are only marginally significant $(p=0.07)$. No significant effects of steroids were found in the remaining hypothalamic nuclei (anterior hypothalamus, Arc, and VMH) $(p>0.4)$.

Finally, the thalamic PIL displayed significant inter-group differences $\left(\chi^{2}\right.$ $(2)=8.510 ; p=0.014)$, as well as the midbrain VLPAG $\left(\chi^{2}(2)=10.900\right.$; $p=0.004)$ with significantly increased levels of pSTAT5-ir relative to control in 
the EP group for the PIL $(p=0.015)$ and in both the EO $(p=0.007)$ and the EP $(p=0.024)$ groups for the VLPAG.

\section{Discussion}

The present work is the first functional analysis of the central lactogenic activity in female mice of an outbred strain (CD1). We have studied the variation of lactogenic signalling through different physiological stages, namely in virgin, late-pregnant, and lactating females. In qualitative terms, we have found variable, but relatively reduced patterns of lactogenic signalling in virgin females in contrast to a widespread common pattern in pregnant and lactating females. In quantitative terms, we have found that PRL-like signalling strongly increases during pregnancy and/or lactation as compared to the basal condition (virgin females). In addition, bromocriptine treatment has no significant effect on the pattern of pSTAT5-ir of late-pregnant females or on the density of pSTAT5-ir in the analysed nuclei.

In this section, we first discuss the advantages and limitations of our methodology. Second, we briefly analyse the expression of pSTAT5 in the brain of virgin females and comment on the effect of gonadal steroids on lactogenic signalling as a possible cause of the variability found in the brain of freely cycling females. Then, we discuss how lactogenic signalling changes with pregnancy and lactation in the analysed regions and which lactogenic agents may be responsible for those changes. Finally, we review the implications of our findings in the current understanding of the mechanisms underpinning maternal behaviour regulation.

\section{Immunohistochemical detection of pSTAT5 as a measure of central PRL receptor signalling}

The methodological approach chosen for this study is the immunohistochemical detection of pSTAT5, which reveals the major lactogenic signalling pathway through the PRLR-1 (mediating actions of PRL and PLs, Soares et al. 1998). However, this methodology does not show signalling independent of STAT5 phosphorylation (Buonfiglio et al. 2015), such as that occurring through the PRLR-s forms (Goupille et al. 1997; Binart et al. 2010). However, evidence suggests that PRLR-s are not functional, or do not relate to the major endocrine actions of PRL (Lesueur et al. 1991). Instead, other studies conclude that the short variants of the PRLR represent a dominant negative form of the receptor, which by dimerization inhibits the functional PRLR-1 (Berlanga et al. 1997). Furthermore, expression levels of the PRLR-s remain unchanged during the estrous cycle, and are similar in virgin, pregnant, and lactating female rats (Sugiyama et al. 1994; Nogami et al. 2007). This supports the view that the 
PRLR-s variants are not directly involved in reproduction. This is one of the reasons why we (and others) focus in the signalling cascade of the long form of the PRLR for analysing the putative role of PRL in maternal physiology and behaviour. Therefore, we have employed the immunohistochemical detection of pSTAT5 to highlight lactogenic signalling through the PRLR-1.

A further concern about this methodology is the potential crosstalk between PRL and growth hormone $(\mathrm{GH})$ or leptin signalling. Both hormones are associated, along with PRL, to receptors of the type-I cytokine receptor superfamily and appear to signal through STAT5, among other pathways (Bennett et al. 2005; Gong et al. 2007). Our observations (see online resources) are in agreement with the previous studies showing that inhibition of hypophyseal PRL release by bromocriptine effectively diminishes pSTAT5 expression within the brain of virgin female mice (Brown et al. 2010) and of lactating dams (Brown et al. 2011). Thus, pSTAT5 immunoreactivity observed in this group reveals signalling exclusively through the PRLR-1. Still, GH (Furigo et al. 2016) or leptin could contribute to the pSTAT5-ir observed in pregnant females, although the high levels of PLs likely account for most (if not all) of the pSTAT5 immunolabelling observed. Regarding leptin, STAT3 is the main STAT member associated with the leptin receptor (Ladyman et al. 2012), whereas STAT5 phosphorylation associated with leptin signalling has been reported only in the arcuate nucleus (Gong et al. 2007; Mütze et al. 2007) and has not been replicated in all studies (Vaisse et al. 1996). As for GH, regions of the brain showing high levels of expression of GH receptors and responsiveness to GH (detected by pSTAT5-ir; Furigo et al. 2016), such as the hippocampus and dentate gyrus (Burton et al. 1992) or layers 2, 3, and 5, and especially, layer 6 of the cerebral cortex (Lobie et al. 1993), show no pSTAT5 labelling in any of our mice, neither in virgin nor in pregnant or lactating females. This suggests that GH signalling in the brain is taking place through alternative pathways, e.g., those involving Src kinases (Waters 2015).

Altogether, pSTAT5 immunostaining can be considered a reliable marker for the activation of the PRLR-1, which, in turn, represents the major component of lactogenic signalling in the brain.

\section{Prolactin signalling in the brain of virgin female mice}

The distribution of pSTAT5-ir in the brain of the female mouse was first published by Brown et al. (2010), who analysed central pSTAT5-ir after exogenous administration of high doses of PRL in virgin females of the inbred strain C57BL/6J. Remarkably, the pattern of pSTAT5-ir they observed was more constant but relatively reduced as compared to the one reported in the present 
work, carried out in CD1 females without exogenous administration of PRL. In addition, our pSTAT5-ir pattern fits the distribution of the mRNA for the PRLR much closer than the pattern of pSTAT5-ir found in C57BL/6J mice. This finding suggests either a mismatch in the brain of $\mathrm{C} 57 \mathrm{BL} / 6 \mathrm{~J}$ mice between the distribution of the mRNA and the receptor protein or some kind of inhibition of central PRL signalling as compared to the CD1 strain. This might be at the base of some functional and behavioural inter-strain differences, for example, the relatively high anxiety and reduced maternal behaviour displayed by C57 females (Parmigiani et al. 1999).

Still, the pattern of pSTAT5-ir in our sample of virgin females exhibits a considerable qualitative variability (summarized in Fig. 2, but see also Fig. OR2). Part of this variability can be attributed to the fact that CD1, the strain used in this study, is an outbred one, hence, showing more intrinsic variability than the most commonly used C57 inbred strain (see Brown et al. 2010). This variability in basal pSTAT5-ir likely reflects variations in factors, such as the circulating PRL levels, access of PRL to the brain or the expression, or sensitivity of PRL receptors. Circulating PRL has been shown to be under the influence of other physiological factors, such as age (Parkening et al. 1982), circadian rhythms (Sinha et al. 1977), or stress (Torner et al. 2002). This source of variability was minimized using females of the same age (2-3 months), which were perfused during the same time window (11:00-13:00 in a 12:12 light-dark cycle, lights ON at 8:00; see "Materials and methods") and group housed to avoid stress derived from social isolation.

Therefore, the most feasible source of variability in pSTAT5-ir within the group of virgins is related to the circulating levels of gonadal steroids. Progesterone and estradiol are well-documented modulators of PRL signalling, which upregulate PRLR expression during proestrus and oestrus (Sugiyama et al. 1994), increase PRLR mRNA levels in pituitary lactotrophs (Scully et al. 1997), and, consequently, circulating PRL levels (Freeman et al. 2000). To explore this possibility, we conducted a hormonal substitution experiment on ovariectomized virgin female mice and assessed the effect of controlled doses of estradiol or estradiol plus progesterone on the central levels of pSTAT5-ir induced by administration of exogenous PRL that render high, likely supraphysiological levels of circulating PRL, irrespective of the endogenous release of hypohyseal PRL. The results of this analysis reveal a general trend towards an increase of pSTAT5-ir density after estradiol treatment (with or without progesterone). This trend reaches significance in five of the analysed regions, namely: the MePD ( $p$ $0.001)$, the LSV $(p=0.004)$, the AC/ADP $(p=0.01)$, the thalamic PIL $(p=0.014)$, and the VLPAG $(p=0.04)$. Of these, only the LSV and the VLPAG display a significant increase in both the EO and EP groups, whereas the 
increase found in the MePD and the PIL groups corresponds only to the EP group and that found in the AC/ADP corresponds only to the EO group. Altogether, this reflects a rather complex regulation of PRL signalling by gonadal steroids. Our results suggest that, indeed, estradiol exerts a positive modulation of lactogenic signalling. On top of estradiol, progesterone might display either a synergistic action with the former (pSTAT5-ir density in the MePD and PIL only increases significantly when both gonadal steroids are administered together) or no significant effect (in the LSV and the VLPAG the significant increase is maintained in both estradiol-treated groups regardless of progesterone).

$\mathrm{AQ} 4$

Surprisingly, our results do not fit completely the colocalisation of estrogen receptor $\alpha(E R \alpha)$ with pSTAT5-ir, as published by Furigo et al. (2014). According to this study, several sites where we have found no apparent differences in pSTAT5-ir density related to gonadal steroid treatment do express abundant ER $\alpha$ receptors colocalising with pSTAT5-ir, e.g., the AVPe, the medial MPO, the Arc, or the VMHvl. Aside from some possibly relevant methodological differences (different PRL dosage and exposition time, for instance), this apparently contradictory results might have different explanations. On the one hand, it is possible that lactogenic signalling was dissociated from gonadal steroid regulation in some regions, depending on the function of PRL in those regions. For example, in the Arc, it is likely that levels of lactogenic signalling respond directly to circulating PRL levels, as the primary function of this nucleus is the feedback control of PRL secretion. Consistent with this, estradiol and progesterone are known to regulate this function through regulation of angiotensin II receptors (Donadio et al. 2006). On the other hand, estradiol regulation of PRL signalling might not always have a positive valence.

According to the existing literature, estradiol is known to regulate the expression of Suppressor of Cytokine Signalling (SOCS) protein family, responsible for impairing signalling through the PRLR. For example, estradiol enhances the activity of SOCS3 promoter in the mouse (Matthews et al. 2005), and chronic estradiol treatment increases the expression of mRNA for SOCS3 and cytokineinducible SH2-containing protein (CIS) in the rat (Anderson et al. 2008), both of which decrease PRL signalling (Krebs and Hilton 2001). Thus, the final levels of lactogenic signalling that we report might result of the balance of opposed regulatory elements and would not reflect exactly the crosstalk with ER.

In sum, our findings together with those of others suggest that, indeed, the central lactogenic signalling is influenced by gonadal steroid levels, but also that this regulation is complex, anatomically heterogeneous, and dependent on different, possibly opposed factors. 


\section{Significance of prolactin-like signalling in the brain during late pregnancy and lactation}

Despite the variability in pSTAT5-ir found in our sample of virgin females, our results confirm, both at a qualitative (Fig. 2) and quantitative (Fig. 6) level, that pregnancy and lactation are associated with a significant increase in the lactogenic signalling in the brain. Exceptions to this trend among the structures, we have analysed are the AVPe (Fig. 6b), the Arc, and the thalamic PIL (Fig. 6c), with comparable pSTAT5-ir density between virgins and pregnant/lactating females. This finding does not fit the results of the previous studies (Brown et al. 2011), reporting an increase in pSTAT5-ir during lactation and after exogenous PRL administration in the AVPe and Arc of C57 animals (PIL was not analysed). Remarkably, Brown et al. (2011) found no associated increase in PRLR expression in these nuclei in lactating dams, but were able to induce a comparable increase in pSTAT5-ir in virgin females by administering exogenous PRL. Therefore, heightened levels of circulating PRL occurring during the lactation period were likely causing the increase in pSTAT-ir of dams found in this work. These data suggest that the lack of inter-group differences in our animals would be due to virgins of the CD1 strain having relatively high (as compared to C57) basal levels of circulating PRL. Supporting this view, the AVPe and the Arc are located next to circumventricular organs (the VOLT and the ME, respectively), thus having privileged access to circulating PRL (Ganong $2000)$.

As for the region we refer to as PIL, the cell cluster we have quantified corresponds to a discrete population that does not exactly match the PIL proper, but rather extends from the medial border of this nucleus into the adjoining region of the posterior thalamus (Fig. 2i). This work is the first description of such population, clearly defined by pSTAT5 immunostaining (Fig. $4 \mathrm{f}$ ). This region is known to be a relay station for ascending somatosensory information of the ventral region of the body, which allows suckling stimulation to trigger the release of PRL during lactation (Cservenák et al. 2013). Therefore, it is tempting to suggest a role of pSTAT5-ir in this region in feedback regulation of sucklinginduced PRL release through a central modulation of sensitivity-to-suckling stimulation. In this context, however, our results revealing comparable levels of pSTAT5-ir in virgin and late-pregnant females and in lactating dams are surprising. The functional meaning of this finding is unclear, but, maybe, could be related to the peculiar maternal behaviour of mice (as compared to rats), where virgin females display nearly spontaneous maternal care (Stolzenberg and Rissman 2011; Martín-Sánchez et al. 2015b).

The generalised increase in pSTAT5-ir during pregnancy and lactation we report 
in this study lends further support to the major role of lactogenic hormones in adapting the female physiology and behaviour to the demands of motherhood (Grattan et al. 2001).

Importantly, we have observed that some of the nuclei analysed in both experiments display a significant increase in pSTAT5-ir density associated with pregnancy and/or lactation, which is not paralleled by an estradiol-dependent increase in virgin ovariectomised females. These include the CeM, BSTMPM, $\mathrm{MPO}, \mathrm{Pa}, \mathrm{SO}$, and VMHvl, which show enhanced lactogenic signalling specific to pregnancy and lactation, but not by estradiol treatment. This suggests that in these nuclei, the increase in pSTAT5-ir during pregnancy is not related to the high circulating levels of estradiol during mid-to-late pregnancy, but to other factors specific to pregnancy and/or lactation, e.g., high levels of circulating placental lactogens (gestation) or PRL (lactation).

According to our results, 8 out of the 13 nuclei sampled in our analysis displayed a statistically significant increase in pSTAT5-ir density already during pregnancy (CeM; MePD; LSV; BSTMPM; Pa; SO; VMHvl, and VLPAG). Two more regions, the $\mathrm{AC} / \mathrm{ADP}$ and the $\mathrm{MPO}$, underwent a moderate increase during pregnancy, which reached significance during lactation (Fig. 6b). This suggests that the maternal adaptations of the brain mediated by lactogenic agents occur before parturition and, in most cases, are maintained subsequently during lactation. Indeed, in two of the analysed nuclei, CeM and SO (see Fig. 6), pSTAT5-ir density peaked during pregnancy and decreased after parturition, so that, in the case of the CeM, levels of pSTAT5-ir were comparable in dams and virgin females. Therefore, pregnancy rather than post-partum seems the critical period for the lactogenic preparation of the brain for motherhood. In further support to this view, it has been shown that mRNA expression of the long form of the PRLR (which signals through the JAK2/STAT5 pathway) is markedly increased in the brain of female rats at mid- and late-gestation, and these elevated mRNA levels are maintained during the lactation period (Sugiyama et al. 1994).

There are, however, two exceptions to this dynamics of pSTAT5-ir, namely the AC/ADP and the MPO (Fig. 6b). Here, levels of pSTAT5-ir moderately increase during late pregnancy, but increase further after parturition, thus reaching statistical difference with virgins only during the lactation period. This suggests a specific role of these nuclei in the regulation and maintenance of maternal behaviours during lactation (see Tsuneoka et al. 2013; Bridges 2015).

Furthermore, inhibition of hypophyseal release of PRL in pregnant females by means of bromocriptine treatment resulted in no significant decrease in pSTAT5- 
ir density as compared to non-treated pregnant females. Although this treatment does not ensure a complete abolishment of hypophyseal PRL in virgin females (low levels of residual immunostaining remain in some brain nuclei, see "Materials and methods"), the previous findings in the literature report a physiological inhibition of hypophyseal PRL secretion during mid-to-late pregnancy resulting in very low levels of circulating PRL $(\leq 5 \mathrm{ng} / \mathrm{ml})$, both in the mouse (Markoff and Talamantes 1981; Soares 2004) and the rat (Andrews et al. 2001 ), as compared to freely cycling, non-pregnant females, with mean levels of $75.4 \pm 5.5 \mathrm{ng} / \mathrm{ml}$ in virgin female CD1 mice (Parkening et al. 1982) and approximately $20 \pm 5 \mathrm{ng} / \mathrm{ml}$ in rats (Carvalho-Freitas et al. 2007).

Administration of bromocriptine on top of this physiological reduction ensures that most, if not all the pSTAT5-ir found in bromocriptine-treated pregnant females is due to non-hypophyseal lactogenic sources, arguably placental lactogen II (Soares 2004). It is also possible that central neurons producing PRL might also be recruited during pregnancy (Paut-Pagano et al. 1993; Grattan and Kokay 2008). Altogether, our findings strongly support the idea that the tuning of the sociosexual brain for motherhood is initiated before parturition mostly by non-hypophyseal lactogenic sources, likely placental lactogens, and maintained subsequently during lactation by high levels of hypophyseal PRL, as suggested by the inhibitory effect of bromocriptine in the presence of neuronal pSTAT5 in lactating mice (Brown et al. 2011).

This would allow, for example, the lactogenic modulation of the release of oxytocin and vasopressin by the magnocellular neurosecretory cells in the $\mathrm{Pa}$ and SO (Parker et al. 1991; Ghosh and Sladek 1995; Grattan et al. 2001; SirzenZelenskaya et al. 2011), the attenuation of stress response at the level of the $\mathrm{Pa}$ (Torner et al. 2002), or the induction of hyperphagia (Sauvé and Woodside 2000; Augustine et al. 2008) already during pregnancy. One of the major outcomes of this process is the prepartum development of maternal behaviours (Slotnick et al. 1973; Lonstein and Gammie 2002; Gammie 2005; Brunton and Russell 2008).

AQ5

Revisiting the role of lactogenic agents in the expression of maternal behaviours

The results of the present work show that several brain nuclei belonging to the so-called maternal brain (Lonstein and Gammie 2002; Gammie 2005) which display a substantial increase in lactogenic signalling during pregnancy and/or lactation, as compared to the basal condition in virgin females. This suggests a role of PRL as an endocrine modulator of the neural centres of maternal behaviours. 
Current evidence on the role of PRL in the management of maternal care is somewhat controversial. On the one hand, maternal care seems to be expressed with independence of PRL action, as suggested by the quick engagement of virgin female mice, as opposed to rats, in allomaternal care (Martín-Sánchez et al. 2015b). This view fits the results of works reporting spontaneous allomaternal care in mice bearing mutations of the genes for PRL (Horseman et al. 1997) or for STAT5 (Buonfiglio et al. (2015). Accordingly, our results reveal only a partial overlapping between pSTAT5-ir patterns and the putative circuitry for maternal care (see Gammie 2005). In this regard, pSTAT5-ir is present in the MPO, which is seen as the key region directing the expression of maternal behaviours (Bridges et al. 1990) and also plays a leading role in the model for "voluntary proactive maternal responses" proposed by Numan and Woodside (2010). This fits a possible role of PRL on motivational, rather than executive, aspects of maternal care (Hauser and Gandelman 1985).

These findings are, however, difficult to reconcile with the profoundly altered maternal behaviour shown by female mice bearing a mutation of the gene for PRLR (Lucas et al. 1998). Further research is needed to clarify this issue.

In contrast to maternal care, it is still unknown whether maternal behaviours not directed to pups, such as nest building or maternal aggression, require PRL or PLs acting through the JAK/STAT pathway. In fact, and in contrast to maternal care, maternal aggression in mice is observed exclusively in dams. Gonadally intact virgin females, even after prolonged intimate contact with pups, do not attack intruders approaching the nest (Martín-Sánchez et al. 2015a). Therefore, the induction of maternal aggression in mice is likely dependent on endocrine factors involved in pregnancy and/or lactation, which, like in rats or hamsters (Wise and Pryor 1977; Mayer et al. 1990), would include PRL and placental lactogens. Consistent with this possibility, the pattern of pSTAT5-ir found in our pregnant-lactating females resembles very closely the nodes of the circuit for maternal aggression (Lonstein and Gammie 2002; Gammie 2005). This network comprises the lateral septum (Brady and Nauta 1953), the BST (Klampfl et al. 2014), the medial and central amygdala (Bosch and Neumann 2010; Unger et al. 2015), the MPA and MPO in the preoptic hypothalamus, the Pa in the anterior hypothalamus and the VMH in the tuberomammillar hypothalamus (Toth et al. 2010; Lin et al. 2011), the paraventricular thalamus (PV) and the caudal PAG (Lonstein and Stern 1997), the peripeduncular region (Factor et al. 1993 ), and the locus coeruleus (LC) in the midbrain. Indeed, every site in this network included in our quantitative evaluation experiments a significant increase in lactogenic signalling during pregnancy and/or lactation (Fig. 6). 
has been dismissed, according to observations that hypophysectomy (Erskine et al. 1980) or bromocriptine treatment (Mann et al. 1980) conducted post-partum do not disrupt maternal aggression. In addition, Broida et al. (1981) observed no correlation between serum PRL levels and maternal aggression in lactating dams. Instead, pup-derived sensory stimulation of the dams has been proposed as the pivotal factor promoting maternal aggression (Garland and Svare 1988). In this work, we show, however, that the lactogenic modulation of brain function leading to a maternal state occurs mainly during late pregnancy (see above). Therefore, prolonged action of lactogenic agents during pregnancy, and not its acute action after parturition, could promote the onset of maternal aggression. Consistent with this, PRL-induced maternal sensitisation in virgin rats is also dependent on prolonged PRL priming, e.g., continuous administration of exogenous PRL (Loundes and Bridges 1986) or prolonged exposure to estradiol eliciting hypophyseal PRL release (Bridges and Ronsheim 1990), rather than acute PRL action. If this was also the case for maternal aggression in mice, the aforementioned lactogenic suppression strategies (hypophysectomy or bromocriptine treatment) applied post-partum would have a minor effect on nest defence.

In further support of this hypothesis, Mann et al. (1984) reported aggression in pregnant female mice against conspecific intruders already during gestation day 14, when neither pup stimuli nor hypophyseal PRL can play a role, but placental lactogens are already acting on the brain, according to our results. Nonetheless, contact with pups and the correct processing of pup-derived stimuli, such as suckling stimulation conveyed by the PIL (Cservenák et al. 2010, 2013) or chemosignal stimuli processed by the MePD (Pardo-Bellver et al. 2012), may be major factors in the maintenance (rather than the onset) of maternal aggression. In fact, information derived from pup chemosignals and from suckling stimulation may converge in the medial amygdala, since it receives projections from the main and accessory olfactory bulbs and the PIL (Cadiz-Moretti et al. 2016).

In addition, evidence in the rat points to the participation of the neuropeptides vasopressin (AVP) and oxytocin (OXT) in maternal aggression, acting at different brain sites, such as the central amygdala or the BST (Bosch and Neumann 2012; Bosch 2013). In this regard, our results indicate that lactogenic agents are also extensively modulating the centres giving rise to the central AVPergic and OXTergic projections, including not only the paraventricular $(\mathrm{Pa})$ and supraoptic (SO) nuclei, but also the BSTMPI or the AC/ADP region (OteroGarcia et al. 2014, 2015).

Conclusion: lactogenic actions in virgin, pregnant, and lactating mice 
In summary, this work explores the physiological variation of PRL-like signalling in the brain of female mice through different stages of their reproductive cycle. Virgin female mice show variable, but generally low pSTAT5-ir, which is modulated by gonadal steroids. This immunoreactivity shows a dramatic increase with pregnancy, resulting in a widespread pattern that remains mostly unaltered during lactation. Furthermore, inhibition of hypophyseal PRL release in pregnant females by means of bromocriptine treatment has no effect on the levels of PRL-like signalling, thus suggesting that placental lactogens (or centrally produced PRL) are responsible for the greater part of the observed lactogenic signalling. Our work provides evidence that the maternal brain is being extensively shaped by lactogenic agents during pregnancy, prior to delivery and lactation, including regions involved in the management of maternal care and maternal aggression.

\section{Acknowledgements}

This work has been funded by the Spanish MINECO-FEDER (BFU2013-47688P), the Junta de Comunidades de Castilla-La Mancha/FEDER (PEIC11-00454490), and the Universitat Jaume I. This work is part of the Doctoral Thesis of Hugo Salais-López, granted by the FPU (Formación de Profesorado Universitario) programme of the Spanish Ministry of Education and Science.

Compliance with ethical standards

Conflict of interest The authors declare that they have no conflict of interest.

\section{Electronic supplementary material}

Below is the link to the electronic supplementary material.

Supplementary material 1 (PDF $718 \mathrm{~kb})$

\section{References}

Anderson GM, Kieser DC, Steyn FJ, Grattan DR (2008) Hypothalamic prolactin receptor messenger ribonucleic acid levels, prolactin signaling, and hyperprolactinemic inhibition of pulsatile luteinizing hormone secretion are dependent on estradiol. Endocrinology 149:1562-1570. doi:10.1210/en.20070867

Andrews ZB, Kokay IC, Grattan DR (2001) Dissociation of prolactin secretion from tuberoinfundibular dopamine activity in late pregnant rats. 
Endocrinology 142:2719-2724. doi:10.1210/en.142.6.2719

Augustine RA, Ladyman SR, Grattan DR (2008) From feeding one to feeding many: hormone-induced changes in bodyweight homeostasis during pregnancy. J Physiol 586:387-397. doi:10.1113/jphysiol.2007.146316

Ben-Jonathan N, Hnasko R (2001a) Dopamine as a prolactin (PRL) inhibitor. Endocr Rev 22:724-763. doi:10.1210/edrv.22.6.0451

Ben-Jonathan N, Hnasko R (2001b) Dopamine as a prolactin (PRL) inhibitor. Endocr Rev 22:724-763. doi:10.1210/er.22.6.724

Bennett E, McGuinness L, Gevers EF et al (2005) Hypothalamic STAT proteins: regulation of somatostatin neurones by growth hormone via STAT5b. J Neuroendocrinol 17:186-194. doi:10.1111/j.13652826.2005.01296.x

Berlanga JJ, Garcia-Ruiz JP, Perrot-Applanat M et al (1997) The short form of the prolactin (PRL) receptor silences PRL induction of the beta-casein gene promoter. Mol Endocrinol 11:1449-1457. doi:10.1210/mend.11.10.9994

Binart N, Bachelot A, Bouilly J (2010) Impact of prolactin receptor isoforms on reproduction. Trends Endocrinol Metab 21:362-368.

doi:10.1016/j.tem.2010.01.008

Bole-feysot C, Goffin V, Edery M et al (1998) Prolactin (PRL) and its receptor: actions, signal PRL receptor knockout mice. Endocr Rev 19:225268. doi:10.1210/er.19.3.225

Bosch OJ (2013) Maternal aggression in rodents: brain oxytocin and vasopressin mediate pup defence. Philos Trans R Soc Lond B Biol Sci 368:20130085. doi:10.1098/rstb.2013.0085

Bosch OJ, Neumann ID (2010) Vasopressin released within the central amygdala promotes maternal aggression. Eur J Neurosci 31:883-891. doi:10.1111/j.1460-9568.2010.07115.x

Bosch OJ, Neumann ID (2012) Both oxytocin and vasopressin are mediators of maternal care and aggression in rodents: from central release to sites of action. Horm Behav 61:293-303. doi:10.1016/j.yhbeh.2011.11.002

Brady JV, Nauta WJ (1953) Subcortical mechanisms in emotional behavior: 
affective changes following septal forebrain lesions in the albino rat. J Comp Physiol Psychol 46:339-346

Bridges RS (1994) The role of lactogenic hormones in maternal behavior in female rats. Acta Paediatr Suppl 397:33-39

Bridges RS (2015) Neuroendocrine regulation of maternal behavior. Front Neuroendocrinol 36:178-196. doi:10.1016/j.yfrne.2014.11.007

Bridges RS, Ronsheim PM (1990) Prolactin (PRL) regulation of maternal behavior in rats: bromocriptine treatment delays and PRL promotes the rapid onset of behavior. Endocrinology 126:837-848. doi:10.1210/endo-126-2-837

Bridges RS, Numan M, Ronsheim PM et al (1990) Central prolactin infusions stimulate maternal behavior in steroid-treated, nulliparous female rats. Proc Natl Acad Sci USA 87:8003-8007. doi:10.1073/pnas.87.20.8003

Bridges RS, Robertson MC, Shiu RP et al (1996) Endocrine communication between conceptus and mother: placental lactogen stimulation of maternal behavior. Neuroendocrinology 64:57-64

Broida J, Michael SD, Svare B (1981) Plasmin prolactin levels are not related to the initiation, maintenance, and decline of postpartum aggression in mice. Behav Neural Biol 32:121-125

Brown RSE, Kokay IC, Herbison AE, Grattan DR (2010) Distribution of prolactin-responsive neurons in the mouse forebrain. J Comp Neurol 518:92102. doi:10.1002/cne. 22208

Brown RSE, Herbison AE, Grattan DR (2011) Differential changes in responses of hypothalamic and brainstem neuronal populations to prolactin during lactation in the mouse. Biol Reprod 84:826-836. doi:10.1095/biolreprod.110.089185

Brown RSE, Wyatt AK, Herbison RE et al (2015) Prolactin transport into mouse brain is independent of prolactin receptor. FASEB J. doi:10.1096/fj.15276519

Brunton PJ, Russell JA (2008) The expectant brain: adapting for motherhood. Nat Rev Neurosci 9:11-25. doi:10.1038/nrn2280 
signaling is required for maintaining lactation but not for postpartum maternal behaviors in mice. Horm Behav 71:60-68. doi:10.1016/j.yhbeh.2015.04.004

Burton KA, Kabigting EB, Clifton DK, Steiner RA (1992) Growth hormone receptor messenger ribonucleic acid distribution in the adult male rat brain and its colocalization in hypothalamic somatostatin neurons. Endocrinology 131:958-963. doi:10.1210/endo.131.2.1353444

Carvalho-Freitas MIR, Anselmo-Franci JA, Teodorov E et al (2007) Reproductive experience modifies dopaminergic function, serum levels of prolactin, and macrophage activity in female rats. Life Sci 81:128-136. doi:10.1016/j.lfs.2007.04.032

Cservenák M, Bodnár I, Usdin TB et al (2010) Tuberoinfundibular peptide of 39 residues is activated during lactation and participates in the sucklinginduced prolactin release in rat. Endocrinology 151:5830-5840. doi:10.1210/en.2010-0767

Cservenák M, Szabó ÉR, Bodnár I et al (2013) Thalamic neuropeptide mediating the effects of nursing on lactation and maternal motivation. Psychoneuroendocrinology 38:3070-3084.

doi:10.1016/j.psyneuen.2013.09.004

Donadio MVF, Gomes CM, Sagae SC et al (2006) Estradiol and progesterone modulation of angiotensin II receptors in the arcuate nucleus of ovariectomized and lactating rats. Brain Res 1083:103-109. doi:10.1016/j.brainres.2006.02.018

Erskine MS, Barfield RJ, Goldman BD (1980) Postpartum aggression in rats: I. Effects of hypophysectomy. J Comp Physiol Psychol 94:484-494. doi: $10.1037 / \mathrm{h} 0077686$

Factor EM, Mayer AD, Rosenblatt JS (1993) Peripeduncular nucleus lesions in the rat: I. Effects on maternal aggression, lactation, and maternal behavior during pre- and postpartum periods. Behav Neurosci 107:166-185

Fleming AS, Rosenblatt JS (1974) Maternal behavior in the virgin and lactating rat. J Comp Physiol Psychol 86:957-972

Freeman ME, Kanyicska B, Lerant A, Nagy G (2000) Prolactin: structure, function, and regulation of secretion. Physiol Rev 80:1523-1631 
Furigo IC, Kim KW, Nagaishi VS et al (2014) Prolactin-sensitive neurons express estrogen receptor- $\alpha$ and depend on sex hormones for normal responsiveness to prolactin. Brain Res 1566:47-59.

doi:10.1016/j.brainres.2014.04.018

Furigo IC, Metzger M, Teixeira PDS et al (2016) Distribution of growth hormone-responsive cells in the mouse brain. Brain Struct Funct. doi:10.1007/s00429-016-1221-1

Gammie SC (2005) Current models and future directions for understanding the neural circuitries of maternal behaviors in rodents. Behav Cogn Neurosci Rev 4:119-135. doi:10.1177/1534582305281086

Ganong WF (2000) Circumventricular organs: definition and role in the regulation of endocrine and autonomic function. Clin Exp Pharmacol Physiol 27:422-427. doi:10.1046/j.1440-1681.2000.03259.x

Garland M, Svare B (1988) Suckling stimulation modulates the maintenance of postpartum aggression in mice. Physiol Behav 44:301-305

Ghosh R, Sladek CD (1995) Role of prolactin and gonadal steroids in regulation of oxytocin mRNA during lactation. Am J Physiol 269:E76-E84

Gong Y, Ishida-Takahashi R, Villanueva EC et al (2007) The long form of the leptin receptor regulates STAT5 and ribosomal protein S6 via alternate mechanisms. J Biol Chem 282:31019-31027. doi:10.1074/jbc.M702838200

Goupille O, Daniel N, Bignon C et al (1997) Prolactin signal transduction to milk protein genes: carboxy-terminal part of the prolactin receptor and its tyrosine phosphorylation are not obligatory for JAK2 and STAT5 activation. Mol Cell Endocrinol 127:155-169

Grattan DR, Kokay IC (2008) Prolactin: a pleiotropic neuroendocrine hormone. J Neuroendocrinol 20:752-763. doi:10.1111/j.13652826.2008.01736.x

Grattan DR, Pi XJ, Andrews ZB et al (2001) Prolactin receptors in the brain during pregnancy and lactation: implications for behavior. Horm Behav 40:115-124. doi:10.1006/hbeh.2001.1698

Hasen NS, Gammie SC (2009) Trpc2 gene impacts on maternal aggression, accessory olfactory bulb anatomy and brain activity. Genes Brain Behav 
8:639-649. doi:10.1111/j.1601-183X.2009.00511.x

Hauser H, Gandelman R (1985) Lever pressing for pups: evidence for hormonal influence upon maternal behavior of mice. Horm Behav 19:454468

Horseman ND, Zhao W, Montecino-Rodriguez E et al (1997) Defective mammopoiesis, but normal hematopoiesis, in mice with a targeted disruption of the prolactin gene. EMBO J 16:6926-6935. doi:10.1093/emboj/16.23.6926

Klampfl SM, Brunton PJ, Bayerl DS, Bosch OJ (2014) Hypoactivation of CRF receptors, predominantly type 2, in the medial-posterior BNST is vital for adequate maternal behavior in lactating rats. J Neurosci 34:9665-9676. doi:10.1523/JNEUROSCI.4220-13.2014

Krebs DL, Hilton DJ (2001) SOCS proteins: negative regulators of cytokine signaling. Stem Cells 19:378-387. doi:10.1634/stemcells.19-5-378

Ladyman SR, Fieldwick DM, Grattan DR (2012) Suppression of leptininduced hypothalamic JAK/STAT signalling and feeding response during pregnancy in the mouse. Reproduction 144:83-90. doi:10.1530/REP-12-0112

Lesueur L, Edery M, Ali S et al (1991) Comparison of long and short forms of the prolactin receptor on prolactin-induced milk protein gene transcription. Proc Natl Acad Sci USA 88:824-828

Leypold BG, Yu CR, Leinders-Zufall T et al (2002) Altered sexual and social behaviors in trp2 mutant mice. Proc Natl Acad Sci USA 99:6376-6381. doi:10.1073/pnas.082127599

Lin D, Boyle MP, Dollar P et al (2011) Functional identification of an aggression locus in the mouse hypothalamus. Nature 470:221-226. doi:10.1038/nature09736

Lobie PE, García-Aragón J, Lincoln DT et al (1993) Localization and ontogeny of growth hormone receptor gene expression in the central nervous system. Brain Res Dev Brain Res 74:225-233

Lonstein JS, Gammie SC (2002) Sensory, hormonal, and neural control of maternal aggression in laboratory rodents. Neurosci Biobehav Rev 26:869888. doi:10.1016/S0149-7634(02)00087-8 
Lonstein JS, Stern JM (1997) Role of the midbrain periaqueductal gray in maternal nurturance and aggression: c-fos and electrolytic lesion studies in lactating rats. J Neurosci 17:3364-3378

Loundes DD, Bridges RS (1986) Length of prolactin priming differentially affects maternal behavior in female rats. Biol Reprod 34:495-501

Lucas BK, Ormandy CJ, Binart N et al (1998) Null mutation of the prolactin receptor gene produces a defect in maternal behavior. Endocrinology 139:4102-4107. doi:10.1210/endo.139.10.6243

Ma FY, Anderson GM, Gunn TD et al (2005) Prolactin specifically activates signal transducer and activator of transcription $5 \mathrm{~b}$ in neuroendocrine dopaminergic neurons. Endocrinology 146:5112-5119. doi:10.1210/en.20050770

Maeda T, Ikegami H, Sakata M et al (1996) Intraventricular administration of estradiol modulates rat prolactin secretion and synthesis. J Endocrinol Invest 19:586-592. doi:10.1007/BF03349022

Mangurian LP, Walsh RJ, Posner BI (1992) Prolactin enhancement of its own uptake at the choroid plexus. Endocrinology 131:698-702.

doi:10.1210/endo.131.2.1639017

Mann M, Michael SD, Svare B (1980) Ergot drugs suppress plasma prolactin and lactation but not aggression in parturient mice. Horm Behav 14:319-328

Mann MA, Konen C, Svare B (1984) The role of progesterone in pregnancyinduced aggression in mice. Horm Behav 18:140-160

Markoff E, Talamantes F (1981) Serum placental lactogen in mice in relation to day of gestation and number of conceptuses. Biol Reprod 24:846-851. doi:10.1095/biolreprod24.4.846

Martinez-Ferre A, Martinez S (2012) Molecular regionalization of the diencephalon. Front Neurosci 6:73. doi:10.3389/fnins.2012.00073

Martín-Sánchez A, McLean L, Beynon RJ et al (2015a) From sexual attraction to maternal aggression: when pheromones change their behavioural significance. Horm Behav 68C:65-76. doi:10.1016/j.yhbeh.2014.08.007 
Wired for motherhood: induction of maternal care but not maternal aggression in virgin female CD1 mice. Front Behav Neurosci 9:197. doi:10.3389/fnbeh.2015.00197

Matthews J, Almlöf T, Kietz S et al (2005) Estrogen receptor-alpha regulates SOCS-3 expression in human breast cancer cells. Biochem Biophys Res Commun 335:168-174. doi:10.1016/j.bbrc.2005.07.057

Mayer AD, Monroy MA, Rosenblatt JS (1990) Prolonged estrogenprogesterone treatment of nonpregnant ovariectomized rats: factors stimulating home-cage and maternal aggression and short-latency maternal behavior. Horm Behav 24:342-364

Mütze J, Roth J, Gerstberger R, Hübschle T (2007) Nuclear translocation of the transcription factor STAT5 in the rat brain after systemic leptin administration. Neurosci Lett 417:286-291. doi:10.1016/j.neulet.2007.02.074

Newman SW (1999) The medial extended amygdala in male reproductive behavior. A node in the mammalian social behavior network. Ann N Y Acad Sci 877:242-257. doi:10.1111/j.1749-6632.1999.tb09271.x

Nogami H, Hoshino R, Ogasawara K et al (2007) Region-specific expression and hormonal regulation of the first exon variants of rat prolactin receptor mRNA in rat brain and anterior pituitary gland. J Neuroendocrinol 19:583593. doi:10.1111/j.1365-2826.2007.01565.x

Numan M, Insel TR (2003) The Neurobiology of Parental Behavior. Springer, New York

Numan M, Woodside B (2010) Maternity: neural mechanisms, motivational processes, and physiological adaptations. Behav Neurosci 124:715-741. doi:10.1037/a0021548

Otero-Garcia M, Martin-Sanchez A, Fortes-Marco L et al (2014) Extending the socio-sexual brain: arginine-vasopressin immunoreactive circuits in the telencephalon of mice. Brain Struct Funct 219:1055-1081.

doi:10.1007/s00429-013-0553-3

Otero-García M, Agustín-Pavón C, Lanuza E, Martínez-García F (2015)

Distribution of oxytocin and co-localization with arginine vasopressin in the brain of mice. Brain Struct Funct. doi:10.1007/s00429-015-1111-y 
Pardo-Bellver C, Cádiz-Moretti B, Novejarque A et al (2012) Differential efferent projections of the anterior, posteroventral, and posterodorsal subdivisions of the medial amygdala in mice. Front Neuroanat 6:1-26. doi:10.3389/fnana.2012.00033

Parkening TA, Collins TJ, Smith ER (1982) Plasma and pituitary concentrations of luteinizing hormone, follicle-stimulating hormone and prolactin in aged, ovariectomized CD-1 and C57BL/6 mice. Exp Gerontol $17: 437-443$

Parker SL, Armstrong WE, Sladek CD et al (1991) Prolactin stimulates the release of oxytocin in lactating rats: evidence for a physiological role via an action at the neural lobe. Neuroendocrinology 53:503-510

Parmigiani S, Palanza P, Rodgers J, Ferrari PF (1999) Selection, evolution of behavior and animal models in behavioral neuroscience. Neurosci Biobehav Rev 23:957-970. doi:10.1016/S0149-7634(99)00029-9

Paut-Pagano L, Roky R, Valatx JL et al (1993) Anatomical distribution of prolactin-like immunoreactivity in the rat brain. Neuroendocrinology 58:682695

Paxinos G, Franklin KBJ (2004) The mouse brain in stereotaxic coordinates. Academic Press, San Diego

Rissman EF, Early AH, Taylor JA et al (1997) Estrogen receptors are essential for female sexual receptivity. Endocrinology 138:507-510. doi:10.1210/endo.138.1.4985

Rood BD, De Vries GJ (2011) Vasopressin innervation of the mouse (Mus musculus) brain and spinal cord. J Comp Neurol 519:2434-2474. doi:10.1002/cne.22635

Sapsford TJ, Kokay IC, Östberg L et al (2012) Differential sensitivity of specific neuronal populations of the rat hypothalamus to prolactin action. $\mathrm{J}$ Comp Neurol 520:1062-1077. doi:10.1002/cne.22775

Sauvé D, Woodside B (2000) Neuroanatomical specificity of prolactininduced hyperphagia in virgin female rats. Brain Res 868:306-314

Scott N, Prigge M, Yizhar O, Kimchi T (2015) A sexually dimorphic hypothalamic circuit controls maternal care and oxytocin secretion. Nature 
Scully KM, Gleiberman AS, Lindzey J et al (1997) Role of estrogen receptoralpha in the anterior pituitary gland. Mol Endocrinol 11:674-681. doi:10.1210/mend.11.6.0019

Sinha YN, Salocks CB, Wickes MA, Vanderlaan WP (1977) Serum and pituitary concentrations of prolactin and growth hormone in mice during a twenty-four hour period. Endocrinology 100:786-791. doi:10.1210/endo-1003-786

Sirzen-Zelenskaya A, Gonzalez-Iglesias AE, Boutet de Monvel J et al (2011) Prolactin induces a hyperpolarising current in rat paraventricular oxytocinergic neurons. J Neuroendocrinol 23:883-893. doi:10.1111/j.13652826.2011.02207.x

Sjoeholm A, Bridges RS, Grattan DR, Anderson GM (2011) Region-, neuron-, and signaling pathway-specific increases in prolactin responsiveness in reproductively experienced female rats. Endocrinology 152:1979-1988. doi:10.1210/en.2010-1220

Soares MJ (2004) The prolactin and growth hormone families: pregnancyspecific hormones/cytokines at the maternal-fetal interface. Reprod Biol Endocrinol 2:51. doi:10.1186/1477-7827-2-51

Soares MJ, Müller H, Orwig KE et al (1998) The uteroplacental prolactin family and pregnancy. Biol Reprod 58:273-284

Stern JM, Kolunie JM (1993) Maternal aggression of rats is impaired by cutaneous anesthesia of the ventral trunk, but not by nipple removal. Physiol Behav 54:861-868

Stolzenberg DS, Rissman EF (2011) Oestrogen-independent, experienceinduced maternal behaviour in female mice. J Neuroendocrinol 23:345-354. doi:10.1111/j.1365-2826.2011.02112.x

Sugiyama T, Minoura H, Kawabe N et al (1994) Preferential expression of long form prolactin receptor mRNA in the rat brain during the oestrous cycle, pregnancy and lactation: hormones involved in its gene expression. $\mathrm{J}$ Endocrinol 141:325-333 
of prolactin in lactation: involvement in behavioural and neuroendocrine stress responses. Eur J Neurosci 15:1381-1389. doi:10.1046/j.14609568.2002.01965.x

Toth M, Fuzesi T, Halasz J et al (2010) Neural inputs of the hypothalamic "aggression area" in the rat. Behav Brain Res 215:7-20.

doi:10.1016/j.bbr.2010.05.050

Tsuneoka Y, Maruyama T, Yoshida S et al (2013) Functional, anatomical, and neurochemical differentiation of medial preoptic area subregions in relation to maternal behavior in the mouse. J Comp Neurol 521:1633-1663. doi:10.1002/cne.23251

Unger EK, Burke KJ, Yang CF et al (2015) Medial amygdalar aromatase neurons regulate aggression in both sexes. Cell Rep 10:453-462. doi:10.1016/j.celrep.2014.12.040

Vaisse C, Halaas JL, Horvath CM et al (1996) Leptin activation of Stat3 in the hypothalamus of wild-type and ob/ob mice but not $\mathrm{db} / \mathrm{db}$ mice. Nat Genet 14:95-97. doi:10.1038/ng0996-95

Wang Z, Storm DR (2011) Maternal behavior is impaired in female mice lacking type 3 adenylyl cyclase. Neuropsychopharmacology 36:772-781. doi:10.1038/npp.2010.211

Waters MJ (2015) The growth hormone receptor. Growth Horm IGF Res. doi:10.1016/j.ghir.2015.06.001

Weiss J, Pyrski M, Jacobi E et al (2011) Loss-of-function mutations in sodium channel Nav1.7 cause anosmia. Nature 472:186-190. doi:10.1038/nature09975

Wise DA, Pryor TL (1977) Effects of ergocornine and prolactin on aggression in the postpartum golden hamster. Horm Behav 8:30-39

Yip SH, Eguchi R, Grattan DR, Bunn SJ (2012) Prolactin signalling in the mouse hypothalamus is primarily mediated by signal transducer and activator of transcription factor 5b but not 5a. J Neuroendocrinol 24:1484-1491. doi:10.1111/j.1365-2826.2012.02357.x 